\title{
Analytical and Sample Preparation Techniques for the Determination of Food Colorants in Food Matrices
}

\author{
Konstantina Ntrallou ${ }^{1}$, Helen Gika ${ }^{2,3}$ (D) and Emmanouil Tsochatzis ${ }^{1,3, *(D)}$ \\ 1 Department of Chemical Engineering, Aristotle University of Thessaloniki, 54124 Thessaloniki, Greece; \\ kondrallou@gmail.com \\ 2 Laboratory of Forensic Medicine \& Toxicology, Department of Medicine, Aristotle University of Thessaloniki, \\ 54124 Thessaloniki, Greece; gkikae@auth.gr \\ 3 BIOMIC AUTH Center for Interdisciplinary Research of the Aristotle University of Thessaloniki, \\ Innovation Area of Thessaloniki, 57001 Thermi, Greece \\ * Correspondence: tsochatzism@gmail.com; Tel.: +30-6977-441091
}

Received: 28 November 2019; Accepted: 3 January 2020; Published: 7 January 2020

\begin{abstract}
Color additives are widely used by the food industry to enhance the appearance, as well as the nutritional properties of a food product. However, some of these substances may pose a potential risk to human health, especially if they are consumed excessively and are regulated, giving great importance to their determination. Several matrix-dependent methods have been developed and applied to determine food colorants, by employing different analytical techniques along with appropriate sample preparation protocols. Major techniques applied for their determination are chromatography with spectophotometricdetectors and spectrophotometry, while sample preparation procedures greatly depend on the food matrix. In this review these methods are presented, covering the advancements of existing methodologies applied over the last decade.
\end{abstract}

Keywords: food colorants (synthetic, natural); food matrices; instrumental analysis; sample preparation

\section{Introduction}

Codex Alimentarius gives a definition for food additives as "any substance that its intentional addition of which to a food aiming for a technological (including organoleptic) purpose in the manufacture, processing, preparation treatment, packing, packaging, transport or holding of such food results, or may be reasonably expected to result, in it or its by-products becoming a component of the food or otherwise affecting the characteristics of such foods" [1,2]. Carocho et al. highlighted that the definition given by the Codex Alimentarius does not include the term contaminants or substances added to food for maintaining or improving nutritional qualities [2].

In food technology, food colorants, of several types, are chemical substances that are added to food matrices, to enhance or sustain the sensory characteristics of the food product, which may be affected or lost during processing or storage, and in order to retain the desired color appearance [3-5]. These are classified based on several criteria: firstly, based on their origin in nature, nature-identical or, if synthetic, whether they are organic or inorganic. Another classification could be based on their solubility (e.g., soluble or insoluble) or covering ability (e.g., transparent or opaque), though an overlap may exist among one or more of these classifications. The most common and widely used classification is based on the distinction between soluble and insoluble color additives (colorants or pigments), which can be further categorized as natural or synthetic [4].

In addition, as described by Martins et al., there were several food additives that had been used extensively in the past but are no longer allowed, due to existing evidence of their side effects, toxicity in the medium- and long-term, as well as a high frequency of potential health incidents [6]. It is also 
important to note that, apart from synthetic food colorants, certain commercial additives of plant or animal origin have also been suspended [3,6-8].

It is clear that the analysis of trace amounts of food colorants is essential with the proper analytical techniques applied, with high specificity and selectivity. Ni et al. has reported that there is increasing interest in the monitoring of the concentration of synthetic food colorants in various products [9].

The analytical methods and sample preparation protocols presented hereafter cover the main techniques that have been applied over the last decade (2008 onwards).

\section{Natural Food Colorants}

Natural additives have been used since ancient times. In certain cases, they were used for the preservation of foodstuffs. Nowadays, most consumers seem to be in favor of the use of the natural, as opposed to the synthetic ones, which are considered by the food industry to be more efficient. In the meantime, there is also considerable interest in the overall reduction of food colorants to food products $[4,5,10]$. The classification of naturally derived colorants can become very complex because of the wide variety of innate properties of the coloring substances. They can be derived from a variety of sources in nature, and therefore, natural colorants also exhibit a wide variety of chemical compositions that affect properties, solubilities, and stabilities differently, and they can have different sources as plant-origin or animal-origin [10].

As reported by Carocho et al., there are benefits linked with the use of natural additives over their respective synthetic ones, which in certain cases present a greater potency over the synthetic ones. The latter in most cases present a single effect on the foodstuff in question. Nevertheless, natural additives are often produced using different methods, i.e., extraction from plants or produced by microorganisms, although there is a tendency to consider them safer than their respective synthetic additive. In general, toxicity is a factor that must be thoroughly assessed and evaluated, to ensure health and safety $[2,5,10]$.

Synthetic colorants have a large span of application and are proportionally lower in cost, than their respective natural substances. However, natural colorants are gradually replacing the synthetic ones as they tend to be considered safer, while presenting higher color specificity, no side effects or related toxicity, and conferring health improving effects and functional benefits to the food itself $[6,11,12]$. A good example for this beneficial effect is the class of yeast-derived natural pigments (e.g., monascin; a yellow natural pigment). These present certain features, apart from food coloring, such as biological activity, reported potential anti-cancer, anti-inflammatory, anti-diabetic, and anti-cholesterolemic effects $[6,13,14]$.

As reported by Martins et al., numerous references highlighted the effective and/or selective use of food colorants. Therefore, for the approved food colorants with an "E" code, individual Acceptable Daily Intakes (ADI) have been approved and established, expressed mostly as mass fractions (i.e., $\mathrm{mg} / \mathrm{kg}$ per body weight (b.w), which can be used for specific purposes (i.e., colorants) in specific food products (i.e., biscuits, chocolates, cheeses etc.) [6].

Commonly, naturally occurring food colorants can be allocated in different sub-categories, namely anthocyanins, carotenoids, beet colorants, and phenolic compounds. In addition, annatto, carminic acid, and some curcuminoids have been studied, particularly curcumin. Finally, other colorants remain to be assessed and evaluated in order to be authorized with an " $E$ " code.

Anthocyanins are a widely studied natural food colorants group, mainly obtained from flowers, fruits, leaves, and even whole plants with a color range that goes from red to purple and blue. Commercial anthocyanins, such as cyanidin 3-glucoside, pelargonidin 3-glucoside, and peonidin 3-glucoside have been used effectively $[2,4,6]$.

Carotenoids are another cluster of naturally derived colorants with a renowned technological effect. They present coloring attributes along with certain bioactive as well as antioxidant properties and are being used extensively in the food industry as natural preservatives $[4,6,7,10,15]$ apart from food colorants [7]. Their main source is extracts from plant roots, flowers, and leaves, as well as 
from algae, yeasts, and aquatic animals. This category mainly includes Lutein, astaxanthin, and lycopene $[2,6]$, the most widely used carotenoids used with others such as crocin and crocetin, still under investigation [4-6].

Red-purple colorants derived from beets and beetroot (Beta vulgaris L.) root is the principal source of these natural colorants but also fruit of Hylocereus polyrhizus (Weber) Britton and Rose, Opuntia ficus-indica [L.] Miller, Opuntia stricta (Haw). Haw and Rivina humilis L. are also rich in these colorant substances, namely, the betacyanins and betalains, which are the most frequently studied and already authorized (E162). They are being used in various food products such as burgers, desserts, ice creams, jams, jellies, soups, sauces, sweets, drinks, dairy products, and yogurts [2,4-6].

Other natural food colorants are considered the phenolic compounds, where flavanones, flavones (4' ,5,7-trihydroxyflavones), and flavonols (fisetin, myricetin, myricitin, quercetin, and rutin) have been studied. As reported by Martins et al., currently only the commercially available products are being used (i.e., myricetin and myricitrin from Myrica cerifera L. roots). Phenolic compounds do not yet have an approved "E" code nor an ADI value [6] with many still being studied and examined since their safety, stability, and spectrum of activity still remain unclear $[6,16]$.

Another category of natural food colorant is the curcuminoids with the most widely known and used colorant in this group being curcumin (E100), usually isolated from Curcuma longa L. rhizomes.

Other natural used colorants are the annatto (E160b) group, as well as bixin and norbixin which are extracts from Bixa orellana L. seeds [2,4-6]. In addition, carminic acid (E120) with a yellow to red-orange food color is already largely used, either naturally occurring or of synthetic origin with an ADI of $5 \mathrm{mg} / \mathrm{kg}$ b.w [6] or crocin. Nevertheless, there are other food colorants under investigation, including c-phycocyanin (blue pigment isolated from Arthrospira platensis) and c-phycoerythrin (red-orange pigment from blue-green algae). Other naturally occurring pigments, which are commercially available, are being studied, such as geniposide, monascorubrin, and purple corn color [4-6].

\section{Synthetic Food Colorants}

Based on increasing demand, mainly from the consumer, for products that are more visually attractive, several synthetic food colorants have been developed for use in food production, to increase certain quality and organoleptic characteristics. However, it is reported that over time, most of the synthetic food colorants were excluded due to repeated side effects as well as to their short- and/or long-term toxicity and eventually to potential carcinogenic effects $[3,6,11]$.

Thus, a change in consumer expectations has been reported, which is largely in favor of the natural colorants $[6,17]$.

Apart from this, also from a regulatory point of view, there is increasing attention and interest related to the risk assessment of these colorants used in food products (i.e., azo-dyes). In case of the azo-dyes, a limiting factor for their use is their potential carcinogenicity, which occurs after their reduction to carcinogenic metabolites into the intestine $[3,18,19]$. These metabolites are produced in the human body, though their toxic effect depends on the ingested amount of the target substance/colorant $[3,18,20]$. However, it is reported that regular evaluation and assessment of potential toxicity of food colorants by regulatory authorities is necessary $[3,18,21]$.

\section{Toxicological Aspects and Regulatory Framework}

Based on various scientific findings, several toxicity effects, have been reported including behavioral effects on children, effects on the respiratory system, connection with allergies, development of attention deficit hyperactivity disorder (ADHD) in children, or neuro-developmental effects at the No-Adverse Effect Limit levels [3,18,21]. In any case, further investigation to assess the potential associated risks of these compounds is needed $[3-9,11,14,18]$.

Several groups have indicated the toxic effect of some of groups of these substances. As an example, Mpountoukas et al. have tested the food colorants amaranth, erythrosine, and tartrazine by in vitro experiments, and they concluded there was an in vitro toxic effect on human lymphocytes 
as they bound to DNA [22]. Many other studies have shown the chemical property of synthetic colorants, namely, Tartrazine [23], azorubine [17,24,25], Allura Red [17,26,27] Sunset Yellow, Quinoline Yellow [17], and Patent Blue [28], to bind to human serum albumin (HSA). Masone and Chanforan compared binding affinities of artificial colorants to human serum albumin (HSA), exhibiting more affinity to HSA than to their natural equivalents' colorants and interacting with its functions. The results supported the hypothesis of their potential risk to human health [17]. Finally, there are dyes, which are rather inexpensive, and which have been used in the food industry, such as Sudan I-IV, which are classified as both a toxic and carcinogenic [24-31]. In Figure 1, basic structures of colorants used in the food industry some of them with toxicological concern (Sudan I-IV) are presented.
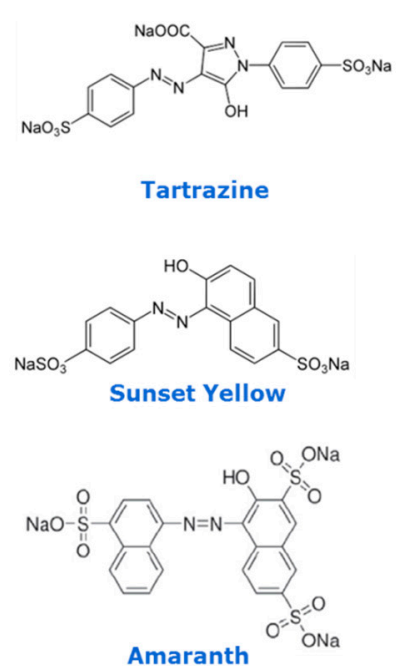

Amaranth
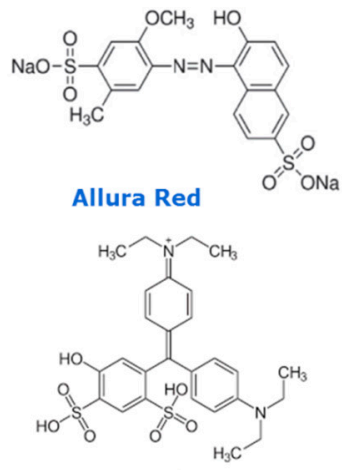

Patent Blue

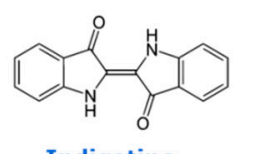

Indigotine

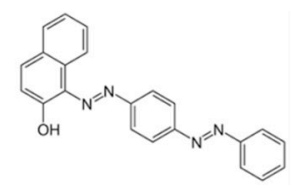

Sudan III
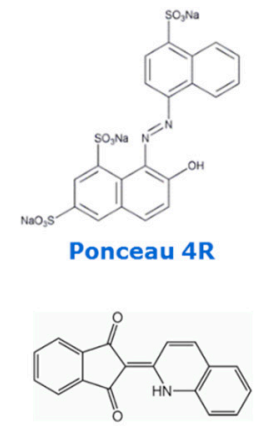

Quinoline yellow
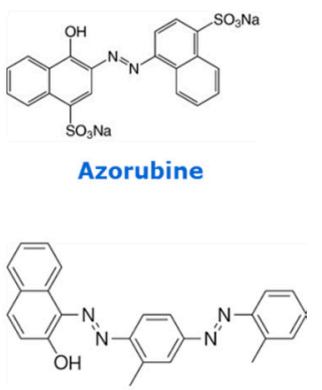

Sudan IV

Figure 1. Chemical structures of selected regulated food colorants.

The main regulatory authorities, EFSA in Europe and the US Food and Drug Administration (FDA) in the United States, are responsible for the evaluation and assessment of food products to enhance and promote health safety $[2,4,5]$. The European Union, set a re-evaluation program of food additives, including food colorants, to be performed by EFSA by 2020, based on the EU Regulation 257/2010. This re-evaluation program was set in order to assess the safety of all authorized food additives in the European Union before 20 January 2009 [32].

The regulatory framework in Europe, in brief, contains the authorization procedure in Regulation (EU) No. 1331/2008, the rules on food additives with a list of approved color additives and their conditions of use in Regulations (EU) No 1333/2008 and 1129/2011, the specifications for food additives in Regulation (EU) 231/2012, and finally for labelling in Regulations (EU) No. 1169/2011 and 1333/2008. Respectively, in the United States, the color additives are included in Title 21 CFR Part 70, listing food additives (exempt from certification, including specifications and conditions of use) in Title 21 CFR Part 73, and certification of donor additives in Title 21 CFR Part $80[4,5,10,33]$.

However, despite the existence of different regulatory frameworks, the overall approach follows similar steps, which are based on well-established risk assessment procedures [33].

Authorization for the use of food colorants in the production of food products is subject to a number of toxicity tests, in order to define and evaluate acute, sub-chronic and chronic toxicity, hepatotoxicity, 
carcinogenicity, mutagenicity, teratotoxicity, genotoxicity, reproductive toxicity, accumulation in the body, bioenergy effects, and immunotoxicity [3-9,11,14,18].

\section{Analytical Methodologies for the Determination of Food Colorants}

\subsection{Analytical Techniques in the Use of Natural Food Colorants Determinations}

The available bibliography concerning the methods of analysis for the natural colorants is limited, compared to that for the synthetic ones, and it is exclusively oriented to their determination in the different naturally deriving products.

All the relative information concerning analytical methods for natural colorants, including tested matrices, analytical technology, type of detection and settings, analytical columns if used, elution parameters, mobile phases, injection volumes, and analytical figures of merit (LOD, LOQ), have been reviewed and are summarized in Table 1.

It can be concluded from Table 1 that evaluation of methods' performance criteria was not within the aims of the above-mentioned reports, as they were focusing in activity, bioavailability, processing impact, and adulteration. Thus, no analytical figures of merit are reported in these papers.

From Table 1 and Figure 2 it could be perceived that the predominant technique is HPLC combined with spectrophotometric (UV-Vis) or Diode Array (DAD) detectors, followed by HPLC by MS/MS. Spectrophotometric UV-Vis methods seem also to be preferred by the researchers in this field as they show low instrument cost and do not involve expert skill. However, it should be considered that the individual features of the spectra obtained for single colors are highly dependent on the $\mathrm{pH}$-adjustment of the solution or the mobile phase, using proper acid or alkali. The $\mathrm{pH}$ adjustment certainly affects maximum absorption wavelength, where shifts and intensities based on the different $\mathrm{pH}$ can be observed. Although sample preparation is much less demanding in comparison to the LC methods, these techniques present a significant disadvantage, which is the lack of ability to analyze simultaneously a bigger number of food colorants.

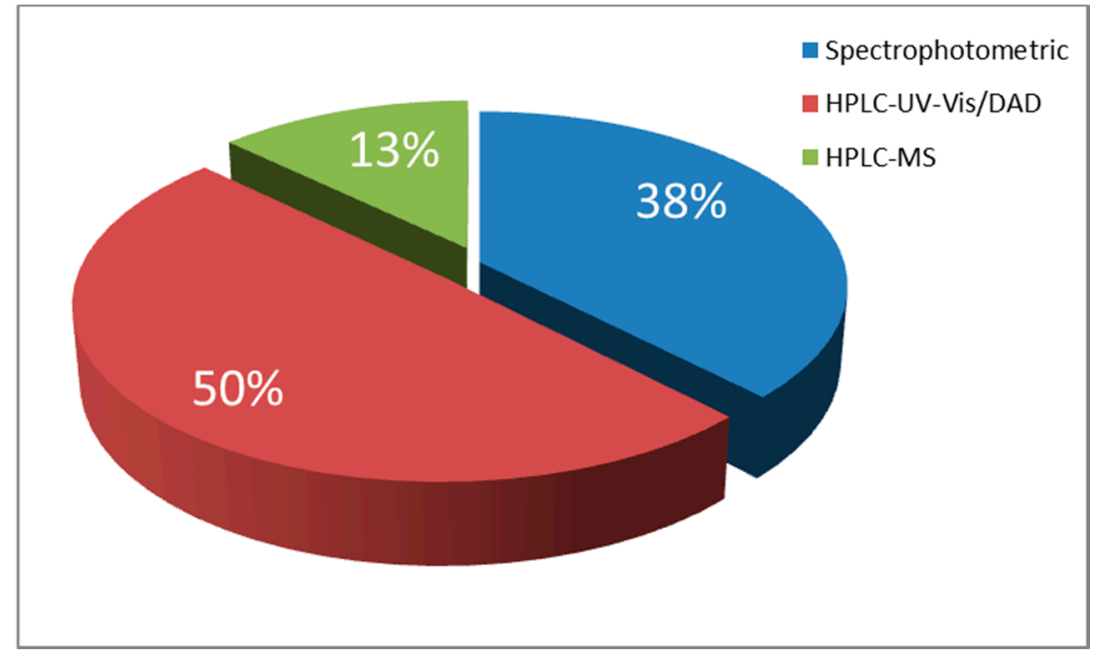

Figure 2. Distribution of techniques used for the analysis of natural food colorants. 
Table 1. Methods for the analysis of natural food colorants in various food products.

\begin{tabular}{|c|c|c|c|c|c|c|c|c|c|c|}
\hline $\begin{array}{c}\text { Food } \\
\text { Colorant }\end{array}$ & $\begin{array}{l}\text { Food } \\
\text { Matrix }\end{array}$ & $\begin{array}{l}\text { Analytical } \\
\text { Technique }\end{array}$ & Detection & $\begin{array}{l}\text { Detection } \\
\text { Settings } \\
\text { (i.e., } \lambda, \\
\text { Ionisation) }\end{array}$ & Column & Elution & Mobile Phase & $\begin{array}{l}\text { Inj. } \\
\text { Volume }\end{array}$ & $\begin{array}{l}\text { Figures of } \\
\text { Merit (LOD, } \\
\text { LOQ, Linear } \\
\text { Range) } \\
\end{array}$ & Ref. \\
\hline $\begin{array}{c}\text { 3-Deoxy- } \\
\text { anthocyanidins }\end{array}$ & $\begin{array}{l}\text { Sorghum bicolor }(\mathrm{L} .) \\
\text { Moench seeds }\end{array}$ & $\begin{array}{l}\text { High Pressure Liquid } \\
\text { Chromato-graphy } \\
\text { (HPLC) }\end{array}$ & $\begin{array}{l}\text { Diode Array } \\
\text { Detection } \\
\text { (DAD) }\end{array}$ & $485 \mathrm{~nm}$ & $\begin{array}{l}\text { Luna C18 column } \\
(150 \times 4.6 \mathrm{~mm}, 5 \mathrm{~mm})\end{array}$ & Gradient & $\begin{array}{l}4 \% \mathrm{HCOOH} \text { in } \mathrm{H}_{2} \mathrm{O}(v / v) \\
\text { (Solvent } \mathrm{A} \text { ) and acetonitrile } \\
\text { (Solvent } \mathrm{B} \text { ) }\end{array}$ & $20 \mu \mathrm{L}$ & $\mathrm{n} / \mathrm{a}$ & [34] \\
\hline \multirow[t]{2}{*}{$\begin{array}{l}\text { Anthocyanin- } \\
\text { derived } \\
\text { extracts }\end{array}$} & $\begin{array}{l}\text { Acacia decurrens } \\
\text { Willd. Bark }\end{array}$ & $\begin{array}{l}\text { Spectrophotometric } \\
\text { analysis }\end{array}$ & UV-Vis & $400-800 \mathrm{~nm}$ & $\mathrm{n} / \mathrm{a}$ & $\mathrm{n} / \mathrm{a}$ & $\mathrm{n} / \mathrm{a}$ & & $\mathrm{n} / \mathrm{a}$ & [35] \\
\hline & -Tulipa gesneriana L. & $\begin{array}{l}\text { Spectrophotometric } \\
\text { analysis }\end{array}$ & UV-Vis & $765 \mathrm{~nm}$ & $\mathrm{n} / \mathrm{a}$ & $\mathrm{n} / \mathrm{a}$ & $\mathrm{n} / \mathrm{a}$ & $\mathrm{n} / \mathrm{a}$ & $\mathrm{n} / \mathrm{a}$ & [36] \\
\hline $\begin{array}{l}\text { Cyanidin } \\
\text { 3-glucoside }\end{array}$ & $\begin{array}{l}\text {-Pistacia lentiscus } \mathrm{L} . \\
\text { fruits; } \\
\text {-Santalum album } \mathrm{L} . \\
\text { fruits }\end{array}$ & HPLC & DAD & $\begin{array}{c}520 \mathrm{~nm}, \\
440 \mathrm{~nm}, \\
310 \mathrm{~nm} \text { and } \\
280 \mathrm{~nm}\end{array}$ & $\begin{array}{c}\text { SS Wakosil C18 } \\
(150 \times 4.6 \mathrm{~mm}, 5 \mu \mathrm{m})\end{array}$ & Gradient & $\begin{array}{l}0.1 \% \text { trifluoroacetic acid } \\
\text { (TFA) in } \mathrm{H}_{2} \mathrm{O} \text { (solvent } \mathrm{A} \text { ) } \\
\text { and } 0.1 \% \text { TFA in } \\
\text { acetonitrile (Solvent B) }\end{array}$ & $20 \mu \mathrm{L}$ & $\mathrm{n} / \mathrm{a}$ & [37] \\
\hline $\begin{array}{l}\text { Cyanidin } \\
\text { 3-glucoside }\end{array}$ & $\begin{array}{l}\text {-Pistacia lentiscus } \mathrm{L} . \\
\text { fruits; } \\
\text {-Santalum album } \mathrm{L} . \\
\text { fruits } \\
\end{array}$ & HPLC & ESI-MS & & $\begin{array}{c}\text { SS Wakosil C18 } \\
(150 \times 4.6 \mathrm{~mm}, 5 \mu \mathrm{m})\end{array}$ & Gradient & $\begin{array}{l}0.1 \% \text { TFA in } \mathrm{H}_{2} \mathrm{O} \text { (solvent } \\
\mathrm{A} \text { ) and } 0.1 \% \text { TFA in } \\
\text { acetonitrile (Solvent } \mathrm{B}\end{array}$ & $20 \mu \mathrm{L}$ & $\mathrm{n} / \mathrm{a}$ & [37] \\
\hline Betacyanins & $\begin{array}{l}\text {-Hylocereus } \\
\text { polyrhizus }\end{array}$ & HPLC & MS & ESI (+) & $\begin{array}{l}\text { AQUA C18-reversed } \\
\text { phase column, } 5 \mu \mathrm{m}\end{array}$ & Gradient & $\begin{array}{c}\text { (A) } 2 \%(v / v) \mathrm{CH}_{3} \mathrm{COOH} \text { in } \\
\mathrm{H}_{2} \mathrm{O} \text { and }(\mathrm{B}) 0.5 \% \\
\mathrm{CH}_{3} \mathrm{COOH} \text { in } \\
\mathrm{H}_{2} \mathrm{O} / \text { acetonitrile }(50 / 50, v / v)\end{array}$ & & $\mathrm{n} / \mathrm{a}$ & [38] \\
\hline Betalains & Beta vulgaris L. roots & HPLC & UV-Vis & $\begin{array}{l}538 \mathrm{~nm} ; \\
480 \mathrm{~nm}\end{array}$ & $\begin{array}{l}\text { Lichrocart } 250 \times 4 \\
\text { RP-18 }(5 \mu \mathrm{m})\end{array}$ & Gradient & $\begin{array}{l}\mathrm{H}_{2} \mathrm{O}(\mathrm{A}) \text { and acetonitrile } \\
\text { (B). }\end{array}$ & $20 \mu \mathrm{L}$ & $\mathrm{n} / \mathrm{a}$ & [39] \\
\hline Betalains & $\begin{array}{l}\text { Opuntia ficus-indica } \\
\text { [L.] }\end{array}$ & HPLC & UV-Vis & $245 \mathrm{~nm}$ & $\begin{array}{l}\text { Luna C18(2) column } \\
(250 \times 4.6 \mathrm{~mm}, 5 \mu \mathrm{m})\end{array}$ & Isocratic & $\begin{array}{c}20 \mathrm{mM} \mathrm{KH}_{2} \mathrm{PO}_{4} / \\
\text { Acetonitrile } 95: 5 v / v\end{array}$ & $20 \mu \mathrm{L}$ & $\mathrm{n} / \mathrm{a}$ & [40] \\
\hline Betalains & $\begin{array}{l}\text { Opuntia ficus-indica } \\
\text { [L.] }\end{array}$ & $\begin{array}{l}\text { Spectrophotometric } \\
\text { analysis }\end{array}$ & UV-Vis & $\lambda=536 \mathrm{~nm}$ & $\mathrm{n} / \mathrm{a}$ & $\mathrm{n} / \mathrm{a}$ & $\mathrm{n} / \mathrm{a}$ & $\mathrm{n} / \mathrm{a}$ & $\mathrm{n} / \mathrm{a}$ & [41] \\
\hline Betalains & $\begin{array}{l}\text { Rivina humilis L. } \\
\text { fruits, juice }\end{array}$ & $\begin{array}{l}\text { Spectrophotometric } \\
\text { analysis }\end{array}$ & UV-Vis & $\lambda=535 \mathrm{~nm}$ & $\mathrm{n} / \mathrm{a}$ & $\mathrm{n} / \mathrm{a}$ & $\mathrm{n} / \mathrm{a}$ & $\mathrm{n} / \mathrm{a}$ & $\mathrm{n} / \mathrm{a}$ & [42] \\
\hline$\alpha$-carotene & $\begin{array}{l}\text { Daucus carota } \mathrm{L} . \\
\text { roots }\end{array}$ & HPLC & UV-Vis & $450 \mathrm{~nm}$ & $\begin{array}{l}\text { Supelcosil LC-18 } \\
\text { column } \\
(15 \mathrm{~cm} \times 4.6 \mathrm{~cm}, 5 \mu \mathrm{m})\end{array}$ & Isocratic & $\begin{array}{l}\text { Methanol/10\% }(v / v) \\
\text { Acetonitrile: } \mathrm{H}_{2} \mathrm{O}\end{array}$ & $50 \mu \mathrm{L}$ & $\mathrm{n} / \mathrm{a}$ & [43] \\
\hline Lutein & Commercial/Milk & HPLC & UV-Vis & $450 \mathrm{~nm}$ & $\begin{array}{c}\text { RP C30 YMC } \\
(250 \times 4.6 \mathrm{~mm}, 5 \mu \mathrm{m})\end{array}$ & Isocratic & $\begin{array}{c}\text { ethanol, } \\
\text { tert-butyl-methyl-ether } \\
\text { (MTBE) as the mobile phase }\end{array}$ & $50 \mu \mathrm{L}$ & n/a & [44] \\
\hline
\end{tabular}


Table 1. Cont.

\begin{tabular}{|c|c|c|c|c|c|c|c|c|c|c|}
\hline $\begin{array}{c}\text { Food } \\
\text { Colorant }\end{array}$ & $\begin{array}{l}\text { Food } \\
\text { Matrix }\end{array}$ & $\begin{array}{l}\text { Analytical } \\
\text { Technique }\end{array}$ & Detection & $\begin{array}{l}\text { Detection } \\
\text { Settings } \\
\text { (i.e., } \lambda, \\
\text { Ionisation) }\end{array}$ & Column & Elution & Mobile Phase & $\begin{array}{l}\text { Inj. } \\
\text { Volume }\end{array}$ & $\begin{array}{c}\text { Figures of } \\
\text { Merit (LOD, } \\
\text { LOQ, Linear } \\
\text { Range) } \\
\end{array}$ & Ref. \\
\hline Lutein & $\begin{array}{l}\text { Hawaii "T. erecta", } \\
\text { Carmen "T. patula". }\end{array}$ & HPLC & DAD & $450 \mathrm{~nm}$ & $\begin{array}{c}\text { Waters-Spherisorb } \\
\text { column SC-04 } \\
(125 \times 4.0 \mathrm{~mm}, \text { ODS2, } \\
3.0 \mu \mathrm{m})\end{array}$ & Gradient & $\begin{array}{l}\text { (A) Acetonitrile-methanol } \\
(9: 1 v / v):(B) \text { Ethyl acetate }\end{array}$ & $100 \mu \mathrm{L}$ & $\mathrm{n} / \mathrm{a}$ & [45] \\
\hline Astaxanthin & $\begin{array}{l}\text { Microalgae and } \\
\text { yeasts }\end{array}$ & HPLC & DAD & $470 \mathrm{~nm}$ & $\begin{array}{c}\text { Chiralcel OD-RH } \\
\text { column }(5 \mu \mathrm{m}, \\
150 \mathrm{~mm} \times 4.6 \mathrm{~mm})\end{array}$ & & $\begin{array}{l}\text { (A) Acetonitrile and (B) } \\
\text { phosphoric acid (3.5 mM) }\end{array}$ & $\mathrm{n} / \mathrm{a}$ & $\mathrm{n} / \mathrm{a}$ & [46] \\
\hline $\begin{array}{l}\text { Crocetin, } \\
\text { Crocin }\end{array}$ & $\begin{array}{l}\text { Grape seed, } \\
\text { monascus, gardenia, } \\
\text { and red radish }\end{array}$ & $\begin{array}{l}\text { Spectrophotometric } \\
\text { analysis }\end{array}$ & UV-Vis & $\begin{array}{l}438 \mathrm{~nm} ; \\
462 \mathrm{~nm}\end{array}$ & $\mathrm{n} / \mathrm{a}$ & $\mathrm{n} / \mathrm{a}$ & $\mathrm{n} / \mathrm{a}$ & $\mathrm{n} / \mathrm{a}$ & $\mathrm{n} / \mathrm{a}$ & [47] \\
\hline $\begin{array}{l}\text { Monascus red } \\
\text { pigments }\end{array}$ & $\begin{array}{l}\text { Beetroot red and } \\
\text { paprika extract }\end{array}$ & $\begin{array}{l}\text { High Resolution Mass } \\
\text { Spectrometry }\end{array}$ & HPLC-QTOF-MS & S ESI (+) & $\begin{array}{l}\text { Kinetex c18 column } \\
(2.6 \mu \mathrm{m}, 50 \mathrm{~mm} \times 4.6 \\
\mathrm{mm})\end{array}$ & Gradient & $\begin{array}{c}\text { (A) Acetonitrile; } \\
\text { (B) } \mathrm{H}_{2} \mathrm{O} ; \\
\text { (C) aqueous } \mathrm{HCOOH} 1 \% \\
v / v\end{array}$ & $\mathrm{n} / \mathrm{a}$ & $\mathrm{n} / \mathrm{a}$ & [48] \\
\hline
\end{tabular}




\subsection{Sample Preparation for Natural Colorant Analysis}

Several sample preparation protocols are reported in the literature by applying various techniques. The applied protocol is strongly dependent by the type and nature of the food sample. Below in Table 2, a short description of the sample preparation protocols is given, along with their application for the clean-up of food samples, for the quantification of natural food colorants. A hydrolysis step with a deprotonation step (ethanol, $\mathrm{HCl}$ solution) is being reported depending on the food matrix, including dilution methods and SFE with supercritical $\mathrm{CO}_{2}$.

Table 2. Sample preparation techniques for the analysis of natural food colorants in food products.

\begin{tabular}{|c|c|c|}
\hline Food Colorant & Extraction/Sample Preparation & Ref. \\
\hline 3-Deoxyanthocyanidins & Ground sample, with $1 \% \mathrm{HCl}$ in methanol, centrifugation hydrolysis; & [34] \\
\hline \multirow[t]{2}{*}{$\begin{array}{l}\text { Anthocyanin-derived } \\
\text { extracts }\end{array}$} & $\begin{array}{c}\text { Comparison of different extraction methods (ultrasonic and natural } \\
\text { extraction) vs. magnetic stirring }\end{array}$ & [35] \\
\hline & Extraction with ethanol: $\mathrm{H}_{2} \mathrm{O}(1: 1 v / v)$ acidified with $0.01 \% \mathrm{HCl}$ & [36] \\
\hline Cyanidin 3-glucoside & $\begin{array}{c}\text { Extraction with } 0.1 \% \mathrm{HCl}(v / v) \text { in methanol, combination of the extracts, } \\
\text { evaporation, and dissolution }\end{array}$ & [37] \\
\hline Betacyanins & $\begin{array}{l}\text { Mixing with water, filtration, addition of ethanol (precipitation of pectic } \\
\text { substances and proteins) }\end{array}$ & [38] \\
\hline Betalains & Sample dissolution in ethanol, agitated and homogenized & [39] \\
\hline Betalains & Filtration of water extract (no $\mathrm{pH}$ adjustment) & [40] \\
\hline Betalains & $\begin{array}{l}\text { Lyophilization and macerated with PBS (pH 5.0) in 1:5 w/w ratio, } \\
\text { followed by spray-drying }\end{array}$ & [41] \\
\hline Betalains & Dilution of the juice; filtration; addition of $\mathrm{Se}^{4+}, \mathrm{Zn}^{2+}$, and $\mathrm{Cu}^{2+}$ & [42] \\
\hline$\alpha$-carotene & $\begin{array}{c}\text { Comparison between simple extraction and Supercritical Fluid } \\
\text { extraction }\left(\mathrm{CO}_{2}\right) ; \\
\text { Simple extraction: Hexane/acetone; SFE: SC-CO } \mathrm{CO}_{2} \text { (SFE) }\end{array}$ & [43] \\
\hline Lutein & $\begin{array}{l}\text { Sample dilution in } 95 \% \text { ethanol and extraction with acetone and } \\
\text { petroleum ether. Evaporation and reconstitution }\end{array}$ & [44] \\
\hline Lutein & $\begin{array}{l}\text { Extraction with organic solvent (isopropanol), centrifugation and } \\
\text { supernatant extracted with hexane }\end{array}$ & [45] \\
\hline Astaxanthin & Extraction with ethyl acetate, filtration & [46] \\
\hline Crocetin, Crocin & Dilution in DMSO & [47] \\
\hline
\end{tabular}

\subsection{Analytical Techniques in the Use of Synthetic Food Colorants Determinations}

The need to determine synthetic colorants in food matrices originating from their known toxicity, renders the analytical task even more challenging as food matrices are ordinarily very complex. Various analytical techniques are used to determine synthetic food colorants in food samples, including spectrophotometry, thin layer chromatography, capillary electrophoresis, high performance liquid chromatography and mass spectrometry (MS).

Certain chemical properties and characteristics of the substances/colorants that influence their separation, such as hydrophilicity/hydrophobicity, existence of acidic or alkaline groups should to be taken into account. Using a Reversed Phase (RP) liquid chromatography separation, more polar compounds are eluting first followed by the less polar. However, their chromatographic separation is normally performed at neutral $\mathrm{pH}$ (ca. 7), and thus, any presence of acidic or alkaline groups could affect the elution sequence.

Ordinarily, organic solvents such as methanol, acetonitrile, or their mixture are used for analysis by HPLC. The addition of acetonitrile improves significantly chromatographic peaks' shape (i.e., asymmetry). Nevertheless, the addition of an inorganic electrolyte as a chemical modifier to the mobile phase can be considered as important in order to advance the separation of all the ionizable species $[12,28,37,49]$. 
Food colorants are compounds that absorb exceedingly in the visible region. Thus, spectrophotometry is sufficient and appropriate for their quantitative analysis. It is generally preferred as a quite straightforward technique, with respective low instrumental cost (i.e., compared to MS/MS). However, in several cases, its main drawback is the lack of specificity, as in case of mixtures of absorbing species. A solution to overcome the problem of specificity is the application of mass spectrometry (MS). In this case, all spectral interventions or interferences, presented on UV-Vis/DAD detectors, are overpassed. High analytical sensitivity could succeed, even in more difficult food matrices, though after proper clean-up. In addition, tandem MS technique could provide structural information based on the molecular mass/ion and the respective fragmentation pattern. Regarding the ionization mode, in most cases, for synthetic colorants, the electro spray ionization (ESI) is preferred because synthetic food colorants are polar molecules, and their ionization efficiency depends on the existence of matrix interferences, present in sample or in the mobile phase. In general, negative mode (ESI-) is more effective, though in other non-regulated substances (i.e., Sudan I-IV) the positive ionization is preferred. During the MS/MS analysis, chemical modifiers (i.e., $\mathrm{HCOONH}_{4}$ or $\mathrm{CH}_{3} \mathrm{COONH}_{4}$ ) are added to the mobile phases, in order to improve and facilitate the better ionization of each target analyte.

Capillary electrophoresis follows in frequency of use the HPLC-DAD/UV-Vis or MS/MS techniques, applied for the quantification of food colorants. These methods present good separation of both small and large molecules, using high voltages. Other reported techniques are FIA (Flow Injection Analysis) and TLC (Thin Layer Chromatography). These could be considered as relatively simple analytical techniques, even for quantification, though in certain cases they could lack specificity and could be affected by matrix interferences.

For synthetic food colorants, all the respective references containing details about the tested matrices, analytical techniques, detection and settings, analytical columns if used, elution, mobile phases, injection volumes, and figures of merit (LOD, LOQ) are presented below in Table 3.

As it could be extrapolated from Table 3, a significant number of LC-MS, LC-MS/MS or LC-UV/Vis methods are available, which are dedicated to simultaneous detection of either a significant or limited number of artificial colorants (whether authorized or delisted), even including illegal Sudan-type dyes. In addition, to Table 3, Figure 3 gives the percentage distribution of the analytical techniques, regarding the analysis of synthetic food colorants. It could be easily concluded that HPLC/U(H)PLC is the most frequently applied technique, followed by capillary electrophoresis and enzyme-linked immunosorbent assay (ELISA) as well as other residual methods. In the case of ELISA, it needs to be highlighted that it cannot be applied for a group of substances/food colorants but only for standalone substances, for which the monoclonal antibodies have been developed.

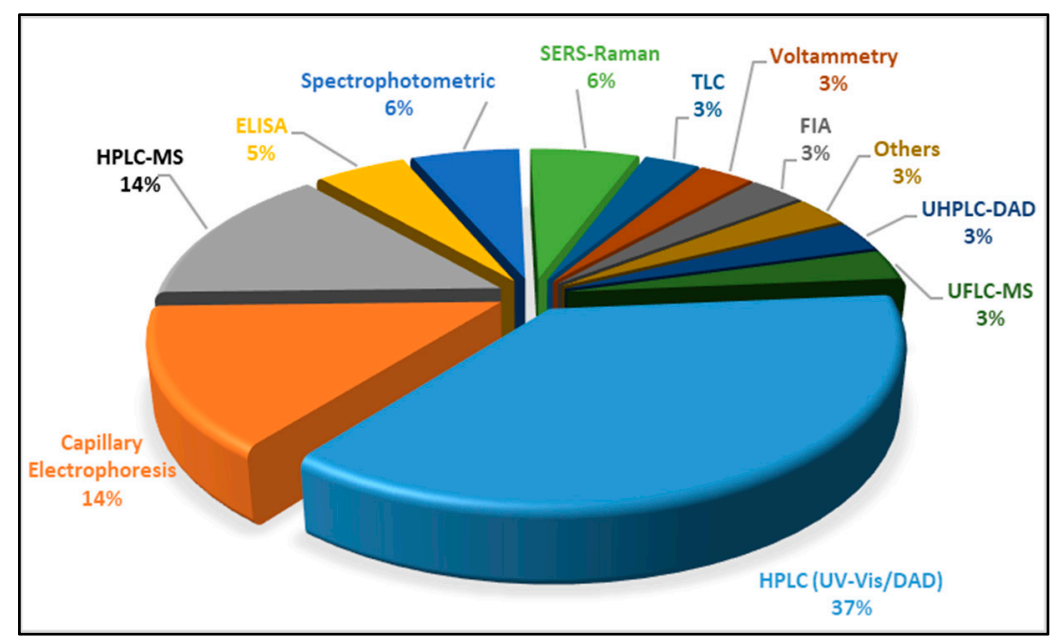

Figure 3. Distribution of techniques for the analysis of synthetic food colorants. 
Table 3. Analytical techniques for the determination of natural food colorants in food samples.

\begin{tabular}{|c|c|c|c|c|c|c|c|c|c|}
\hline $\begin{array}{c}\text { Food } \\
\text { Colorant }\end{array}$ & MATRIX & $\begin{array}{l}\text { Analytical } \\
\text { Technique }\end{array}$ & Detection & Column & Elution & Mobile Phase & Inj. Volume & $\begin{array}{l}\text { Figures of Merit } \\
\text { (LOD, LOQ) }\end{array}$ & Ref. \\
\hline Brilliant blue & Liquid foods & CE & $\begin{array}{c}\mathrm{UV}(\lambda=220 \mathrm{~nm}) \\
36 \mathrm{~cm} \text { capillary; } \\
\text { Separation voltage } \\
(8 \mathrm{kV},-8 \mathrm{kV})\end{array}$ & $\begin{array}{c}\text { Fused-silica } \\
\text { cappilaries of } \\
375 \text { od } \mu \mathrm{m} \text { and } \\
75 \mu \mathrm{m} \text { i.d } \\
\end{array}$ & - & $\begin{array}{c}30 \mathrm{mM} \text { PBS buffer (pH6), } \\
\text { with } 0.9 \mathrm{mg} / \mathrm{mL} \text { dASNPs } \\
\text { and } 2 \mathrm{mM} \beta \text {-cyclodextrin } \\
\text { (CD) }\end{array}$ & $\begin{array}{l}\text { Electro kinetic } \\
\text { injection }\end{array}$ & $\begin{array}{l}\mathrm{LOD}=0.36 \mathrm{mg} / \mathrm{L} \\
\mathrm{LOQ}=0.63 \mathrm{mg} / \mathrm{L}\end{array}$ & [2] \\
\hline $\begin{array}{l}\text { Amaranth, ponceau } 4 \mathrm{R} \text {, sunset } \\
\text { yellow, tartrazine, brilliant blue }\end{array}$ & & $\begin{array}{l}\text { spectrophotometric } \\
\text { kinetic } \\
\text { method }\end{array}$ & $\begin{array}{c}\text { UV-Vis } \\
\lambda_{\text {Prussian blue }}=760 \mathrm{~nm}\end{array}$ & $\mathrm{n} / \mathrm{a}$ & $\mathrm{n} / \mathrm{a}$ & $\mathrm{n} / \mathrm{a}$ & $\mathrm{n} / \mathrm{a}$ & $\mathrm{LOD}=0.2-6.0 \mathrm{mg} / \mathrm{L}$ & [9] \\
\hline Sudan I & $\begin{array}{l}\text { Non-alcoholic } \\
\text { drinks, sweets, } \\
\text { jellies }\end{array}$ & $\begin{array}{l}\text { Enzyme-linked } \\
\text { Immuno-sorbent } \\
\text { assay } \\
\text { (ELISA) }\end{array}$ & $\mathrm{n} / \mathrm{a}$ & $\mathrm{n} / \mathrm{a}$ & $\mathrm{n} / \mathrm{a}$ & $\mathrm{n} / \mathrm{a}$ & $\mathrm{n} / \mathrm{a}$ & $\mathrm{LOD}=0.07 \mathrm{ng} / \mathrm{mL}$ & [29] \\
\hline Sudan I & $\begin{array}{l}\text { Non-alcoholic } \\
\text { drinks, jellies }\end{array}$ & HPLC & $\begin{array}{l}\text { UV- Vis } \\
(478 \mathrm{~nm})\end{array}$ & $\begin{array}{c}\mathrm{C}_{18}(250 \times 4.6 \mathrm{~mm}, \\
5.0 \mu \mathrm{m})\end{array}$ & Gradient & methanol/2\% $\mathrm{CH}_{3} \mathrm{COOH}$ & $20 \mu \mathrm{L}$ & $\mathrm{LOD}=0.14 \mathrm{ng} / \mathrm{mL}$ & [29] \\
\hline $\begin{array}{c}\text { Tartrazine, quinoline Yellow, } \\
\text { sunset yellow, Carmoisine, } \\
\text { Amaranth, ponceau 4R, } \\
\text { Erythrosine, Red } \\
\text { 2G, allura Red AC, Patent Blue V, } \\
\text { Indigo Carmine, brilliant blue, } \\
\text { Green S }\end{array}$ & $\begin{array}{l}\text { Beverages, dairy } \\
\text { powders, jellies, } \\
\text { candies, } \\
\text { condiments, } \\
\text { icings, syrups, } \\
\text { extracts }\end{array}$ & HPLC & $\begin{array}{c}\text { DAD } \\
\text { Various wavelengths }\end{array}$ & $\begin{array}{c}\text { Discovery C18 } \\
(250 \mathrm{~mm} \times 4.6 \mathrm{~mm} \\
5 \mu \mathrm{m})\end{array}$ & Gradient & $\begin{array}{c}\mathrm{CH}_{3} \mathrm{COONH}_{4} 0.13 \mathrm{M} \\
(\mathrm{pH}=7.5 ; \\
\mathrm{NaOH}) / \text { methanol: } \\
\text { acetonitrile } 80: 20 \mathrm{v} / \mathrm{v}\end{array}$ & $20 \mu \mathrm{L}$ & $\mathrm{LOD}=1.87-22.1 \mu \mathrm{g} / \mathrm{L}$ & [49] \\
\hline $\begin{array}{l}\text { Tartrazine, sunset yellow, } \\
\text { brilliant Blue, acid red }\end{array}$ & Powder & SERS-Raman & $\begin{array}{l}\text { confocal microscope } \\
\text { Raman spectrometer } \\
\text { system }\end{array}$ & $\mathrm{n} / \mathrm{a}$ & $\mathrm{n} / \mathrm{a}$ & $\mathrm{n} / \mathrm{a}$ & $\mathrm{n} / \mathrm{a}$ & $\mathrm{LOD}=10^{-7} \mathrm{M}$ & [50] \\
\hline $\begin{array}{l}\text { Allura red, sunset yellow, } \\
\text { tartrazine }\end{array}$ & Soft drinks & HPLC & DAD & $\mathrm{n} / \mathrm{a}$ & Gradient & $\begin{array}{c}\text { methanol (HPLC grade) } \\
\text { and } \mathrm{NaH}_{2} \mathrm{PO}_{4} / \\
\mathrm{Na}_{2} \mathrm{HPO}_{4} \text { buffer }(0.10 \mathrm{M}, \\
\mathrm{pH}=7.0) .\end{array}$ & $\mathrm{n} / \mathrm{a}$ & $\begin{array}{c}\mathrm{LOD}=0.06-0.30 \\
\mu \mathrm{g} / \mathrm{mL}\end{array}$ & [51] \\
\hline $\begin{array}{l}\text { Allura red, sunset yellow, } \\
\text { tartrazine }\end{array}$ & Soft drinks & HLA-Go & - & - & - & - & - & - & [51] \\
\hline $\begin{array}{c}\text { Azorubine, amaranth, cochineal } \\
\text { red A, red 2G, allura red, } \\
\text { azocarmine B (AZO B), } \\
\text { azocarmine G (AZO G), ponceau } \\
\text { 2R, ponceau } 6 \mathrm{R}, \text { tartrazine, } \\
\text { sunset yellow, quinoline yellow, } \\
\text { orange II, metanil yellow (MY), } \\
\text { patent blue V, indigo carmine, } \\
\text { brilliant blue }\end{array}$ & $\begin{array}{c}\text { Solid } \\
\text { food/liquid } \\
\text { beverages }\end{array}$ & HPLC & $\begin{array}{c}\text { DAD } \\
\text { DAD, } \lambda_{\text {quant. }} \\
-620 \mathrm{~nm} \text { (blue); } \\
-515 \mathrm{~nm} \text { (red); } \\
-420 \text { and } 480 \mathrm{~nm} \\
\text { (yellow). }\end{array}$ & $\begin{array}{c}\mathrm{C} 8(150 \times 4.6 \mathrm{~mm} \\
3 \mu \mathrm{m})\end{array}$ & Gradient & $\begin{array}{l}\text { Acetonitrile/sodium } \\
\text { acetate }(\mathrm{pH}=7)\end{array}$ & $20 \mu \mathrm{L}$ & $\begin{array}{l}\text { 5-300 mg/kg (solid } \\
\text { food samples) } \\
\text { 5-100 mg/L (drinks) }\end{array}$ & {$[52]$} \\
\hline
\end{tabular}


Table 3. Cont

\begin{tabular}{|c|c|c|c|c|c|c|c|c|c|}
\hline $\begin{array}{c}\text { Food } \\
\text { Colorant }\end{array}$ & MATRIX & $\begin{array}{l}\text { Analytical } \\
\text { Technique }\end{array}$ & Detection & Column & Elution & Mobile Phase & Inj. Volume & $\begin{array}{l}\text { Figures of Merit } \\
\text { (LOD, LOQ) }\end{array}$ & Ref. \\
\hline $\begin{array}{c}\text { Brilliant blue, Tartrazine, } \\
\text { amaranth, carmine, sunset } \\
\text { yellow, } \\
\text { allura red, erythrosine } \\
\end{array}$ & $\begin{array}{l}\text { Wine and soft } \\
\text { drinks }\end{array}$ & UFLC & ESI (-)-MS/MS & $\begin{array}{c}\text { ODS II } \\
(100 \mathrm{~mm} \times 2.0 \mathrm{~mm} ; \\
2.2 \mu \mathrm{m})\end{array}$ & Gradient & $\begin{array}{c}\text { A: Acetonitrile: } \\
\mathrm{CH}_{3} \mathrm{COONH}{ }_{4} 5.0 \mathrm{mM} / \\
\text { (B) } \mathrm{H}_{2} \mathrm{O}: \mathrm{CH}_{3} \mathrm{COONH} \\
\\
5.0 \mathrm{mM}\end{array}$ & $5 \mu \mathrm{L}$ & $\begin{array}{l}\mathrm{LOD}=0.45-1.51 \mu \mathrm{g} / \mathrm{L} \\
\mathrm{LOQ}=1.51-5.00 \mu \mathrm{g} / \mathrm{L}\end{array}$ & [53] \\
\hline $\begin{array}{l}\text { Brilliant blue, tartrazine, } \\
\text { amaranth, sunset yellow }\end{array}$ & $\begin{array}{l}\text { Wine and soft } \\
\text { drinks }\end{array}$ & TLC-UV-Vis & UV-Vis & $\begin{array}{l}\text { TLC-PET } 20 \times 20 \\
\text { silica gel }\end{array}$ & $\mathrm{n} / \mathrm{a}$ & $\begin{array}{c}8 \mathrm{~mL} \text { 2-propanol and } \\
3 \mathrm{~mL} \mathrm{NH}_{4} \mathrm{OH}\end{array}$ & $\begin{array}{c}5 \mu \mathrm{L} \\
\text { (standards) } \\
\text { and } 30 \mu \mathrm{L} \\
\text { sample }\end{array}$ & $\mathrm{n} / \mathrm{a}$ & [54] \\
\hline $\begin{array}{l}\text { Allura red, sunset yellow, } \\
\text { tartrazine }\end{array}$ & $\begin{array}{c}\text { Solid } \\
\text { food/liquid } \\
\text { beverages }\end{array}$ & $\begin{array}{l}\text { Spectrophoto-metric } \\
\text { BLLS/RBL }\end{array}$ & $\begin{array}{c}\text { Absorbance } \\
\text { spectra-pH data } \\
\text { Spectral } \\
\text { measurements } \\
(300-600 \mathrm{~nm}) \text { at } \\
\text { different pH }\end{array}$ & n.a & n.a & n.a & n.a & $\mathrm{LOD}=0.54 \mathrm{mg} / \mathrm{L}$ & [55] \\
\hline Sunset yellow & Beverage & HPLC & ESI (-)-MS & $\begin{array}{c}\text { C18-ether column } \\
(150 \mathrm{~mm} \times 4.6 \mathrm{~mm}, \\
5 \mu \mathrm{m})\end{array}$ & Isocratic & $\begin{array}{l}\text { (A) } 63 \% \text { aqueous } \\
\text { solution } 20 \mathrm{mM} \\
\mathrm{CH}_{3} \mathrm{COONH} \\
\text { (B) } \\
\text { (B) } 37 \% \text { methanol }\end{array}$ & $20 \mu \mathrm{L}$ & $\mathrm{n} / \mathrm{a}$ & [56] \\
\hline Carmoisine, sunset yellow & Beverage & HPLC & ESI (-)-MS & $\begin{array}{l}\text { C18 column }(250 \\
\mathrm{mm} \times 2 \mathrm{~mm}, 4 \mu \mathrm{m})\end{array}$ & Isocratic & $\begin{array}{c}\text { (A) Methanol and (B) } \\
10 \mathrm{mM} \mathrm{HCOONH}_{4} \\
(45: 55, v / v)\end{array}$ & $20 \mu \mathrm{L}$ & $\mathrm{LOD}=10-12 \mu \mathrm{g} / \mathrm{L}$ & [57] \\
\hline Allura red & Beverage & HPLC & ESI (-)-MS & $\begin{array}{c}\text { HSS-T3 } \\
\text { column }(2.1 \mathrm{~mm} \\
100 \mathrm{~mm}, 1.8 \mu \mathrm{m})\end{array}$ & Gradient & $\begin{array}{c}\text { A: } \mathrm{H}_{2} \mathrm{O}: \mathrm{CH}_{3} \mathrm{COONH}_{4} \\
1.0 \mathrm{mM} / \\
\text { (B) Methanol: } \\
\mathrm{CH}_{3} \mathrm{COONH}_{4} 1.0 \mathrm{mM} \\
\end{array}$ & $20 \mu \mathrm{L}$ & $\mathrm{n} / \mathrm{a}$ & {$[58,59]$} \\
\hline $\begin{array}{l}\text { Brilliant blue, tartrazine, allura } \\
\text { red, amaranth, Azorubine, } \\
\text { patent Blue V, ponceau } 4 \mathrm{R}\end{array}$ & $\begin{array}{l}\text { Various food } \\
\text { products }\end{array}$ & HPLC & $\begin{array}{c}\text { DAD } \\
\text { Various wavelengths }\end{array}$ & $\begin{array}{c}\text { Xterra RP18 } \\
\text { column } \\
(250 \times 4.6 \mathrm{~mm} \\
5 \mu \mathrm{m}) \\
\end{array}$ & Gradient & $\begin{array}{c}\text { A) } 0.1 \mathrm{M} \mathrm{CH}_{3} \mathrm{COONH}_{4} \\
\text { in water and (B) } 0.1 \mathrm{M} \\
\mathrm{CH}_{3} \mathrm{COONH} \\
\text { methanol } \\
\end{array}$ & $20 \mu \mathrm{L}$ & $\begin{array}{c}\mathrm{LOD}=0.02-1.49 \\
\mathrm{mg} / \mathrm{L}\end{array}$ & [60] \\
\hline $\begin{array}{c}\text { Brilliant blue, indigo carmine, } \\
\text { allura red, carminic acid, } \\
\text { ponceau } 4 R \text {, sunset yellow, } \\
\text { tartrazine }\end{array}$ & $\begin{array}{c}\text { Dairy powders, } \\
\text { color beverages, } \\
\text { jellies, candies, } \\
\text { condiments, } \\
\text { icings, syrups, }\end{array}$ & $\mathrm{CE}$ & $\begin{array}{c}\text { UV }(200 \mathrm{~nm}) \\
\text { Condiiton with } 1 \mathrm{M} \\
\mathrm{NaOH}, \mathrm{H}_{2} \mathrm{O} \\
\text { electrode polarity } \\
(25 \mathrm{kV})\end{array}$ & - & - & $\begin{array}{l}\text { Running buffer of } \mathrm{pH} 10 \\
(20 \mathrm{mM} \mathrm{NaOH} \text { solution } \\
\text { to } 15 \mathrm{mM} \text { disodium } \\
\text { tetraborate (borax) to } \\
20 \mathrm{mM} \text { NaOH, until the } \\
\text { desired } \mathrm{pH}\end{array}$ & $\begin{array}{l}\text { Large-volume } \\
\text { injection }\end{array}$ & $\begin{array}{l}\text { LODs } 0.05-0.40 \\
\mu \mathrm{g} / \mathrm{mL}\end{array}$ & [61] \\
\hline $\begin{array}{l}\text { Brilliant blue, indigo carmine, } \\
\text { allura red, carminic acid, } \\
\text { ponceau } 4 \mathrm{R} \text {, sunset yellow, } \\
\text { tartrazine, fast green FCF }\end{array}$ & Liquid foods & $\mathrm{CE}$ & $\begin{array}{c}\mathrm{UV}(\lambda=200 \mathrm{~nm}) \\
\text { Condiiton with } 1 \mathrm{M} \\
\mathrm{NaOH}, \mathrm{H}_{2} \mathrm{O} \\
\text { electrode polarity } \\
(25 \mathrm{kV})\end{array}$ & - & & $\begin{array}{l}\text { Running buffer of } \mathrm{pH} 10 \\
\text { (20 mM NaOH to } 15 \mathrm{mM} \\
\text { disodium tetraborate } \\
\text { (borax) to } 20 \mathrm{mM} \mathrm{NaOH} \text {, } \\
\text { until desired } \mathrm{pH}\end{array}$ & $\begin{array}{c}\text { Large-volume } \\
\text { injection }\end{array}$ & $\begin{array}{c}\mathrm{LOD}=0.002-0.026 \\
\mu \mathrm{g} / \mathrm{mL}\end{array}$ & [62] \\
\hline
\end{tabular}


Table 3. Cont

\begin{tabular}{|c|c|c|c|c|c|c|c|c|c|}
\hline $\begin{array}{c}\text { Food } \\
\text { Colorant }\end{array}$ & MATRIX & $\begin{array}{l}\text { Analytical } \\
\text { Technique }\end{array}$ & Detection & Column & Elution & Mobile Phase & Inj. Volume & $\begin{array}{l}\text { Figures of Merit } \\
\text { (LOD, LOQ) }\end{array}$ & Ref. \\
\hline $\begin{array}{l}\text { Sunset yellow, carmoisine, } \\
\text { amaranth, ponceau } 4 \mathrm{R}, \\
\text { erythrosine, red 2G, allura red }\end{array}$ & Soft drinks & HPLC & $\begin{array}{c}\text { DAD } \\
\text { Various wavelengths }\end{array}$ & $\begin{array}{l}\text { Symmetry C18 } \\
\text { (Waters, Milford, } \\
\text { USA) } \\
\text { column }(150 \mathrm{~mm} \times \\
4.6 \mathrm{~mm}, 5 \mu \mathrm{m})\end{array}$ & Gradient & $\begin{array}{c}\mathrm{CH}_{3} \mathrm{COONH}_{4} \\
\text { buffer }(1 \% w / v)(0.13 \mathrm{M}) \\
\text { (pH: } 7.5) \text { by addition of } \\
0.1 \mathrm{M} \\
\text { aq. } \mathrm{NH}_{3}(\text { solvent } \mathrm{A}) \\
\text { methanol (solvent } \mathrm{B}) \text { and } \\
\text { acetonitrile } \\
\text { (solvent } \mathrm{C})\end{array}$ & $\mathrm{n} / \mathrm{a}$ & $0.5-1.4 \mu \mathrm{g} / \mathrm{mL}$ & [63] \\
\hline $\begin{array}{l}\text { Tartrazine, quinoline yellow, } \\
\text { sunset yellow, carmoisine, } \\
\text { ponceau } \\
\text { 4R, allura red, indigo carmine, } \\
\text { brilliant blue }\end{array}$ & $\begin{array}{l}\text { Various foods } \\
\text { and medicines }\end{array}$ & HPLC & $\begin{array}{c}\text { DAD } \\
\text { Various wavelengths }\end{array}$ & $\begin{array}{c}\text { C18 column } \\
(250 \mathrm{~mm} \times 4.6 \mathrm{~mm} \\
5 \mu \mathrm{m})\end{array}$ & Isocratic & $\begin{array}{l}\text { A) Triton X-100 (0.25\%, } \\
v / v) \text { and (B) } 50 \mathrm{mmol} / \mathrm{L} \\
\text { PBS (pH 7) }\end{array}$ & $20 \mu \mathrm{L}$ & $\begin{array}{c}\mathrm{LOD}=0.05-0.44 \\
\mu \mathrm{g} / \mathrm{mL} \\
\mathrm{LOQ}=0.05-1.12 \\
\mu \mathrm{g} / \mathrm{mL}\end{array}$ & [64] \\
\hline $\begin{array}{c}\text { Tartrazine, sunset yellow, } \\
\text { azorubine, amaranth, cochineal } \\
\text { red, red 2G, allura red AC, } \\
\text { Brilliant Black BN, brown FK, } \\
\text { Brown HT } \\
\text { Patent Blue V, brilliant Blue FCF, } \\
\text { Green S } \\
\end{array}$ & Fish roe & HPLC & $\begin{array}{c}\text { DAD } \\
\text { Various wavelengths }\end{array}$ & $\begin{array}{c}\text { Xterra RP18 } \\
\text { column } \\
(250 \times 4.6 \mathrm{~mm} \\
5 \mu \mathrm{m})\end{array}$ & Gradient & $\begin{array}{c}\text { (a) } 100 \mathrm{mmol} / \mathrm{L} \\
\mathrm{CH}_{3} \mathrm{COONa} \text { buffer (pH } \\
\text { 7.0) and (b) Acetonitrile }\end{array}$ & $20 \mu \mathrm{L}$ & $\begin{array}{c}\mathrm{LOD}=0.02-1.49 \\
\mathrm{mg} / \mathrm{L}\end{array}$ & [65] \\
\hline $\begin{array}{l}\text { Brilliant blue, Indigo carmine, } \\
\text { allura red, erythrosine, ponceau } \\
4 \mathrm{R} \text {, sunset yellow, Lemon yellow }\end{array}$ & $\begin{array}{l}\text { Protein-rich } \\
\text { samples }\end{array}$ & HPLC & $\begin{array}{c}\text { DAD } \\
\text { Various wavelengths }\end{array}$ & RP-C18 Column & Gradient & $\begin{array}{l}\text { Methanol- } 20 \mathrm{mM} \text { of } \\
\mathrm{CH}_{3} \mathrm{COONH}\end{array}$ & $20 \mu \mathrm{L}$ & $\mathrm{LOD}=0.1-0.4 \mathrm{mg} / \mathrm{kg}$ & {$[66]$} \\
\hline $\begin{array}{l}\text { Brilliant blue, tartrazine, sunset } \\
\text { yellow, amaranth, carminic acid, } \\
\text { acid red, allura red }\end{array}$ & Meat products & UHPLC & $\begin{array}{c}\text { PDA } \\
\text { Various wavelengths }\end{array}$ & $\begin{array}{c}\text { BEH C18 } \\
(100 \times 2.1 \mathrm{~mm}, \\
1.7 \mu \mathrm{m} \\
\end{array}$ & Gradient & $\begin{array}{l}\text { Acetonitrile/ } \\
\mathrm{CH}_{3} \mathrm{COONH}_{4}\end{array}$ & $5 \mu \mathrm{L}$ & $\begin{array}{l}\mathrm{LOD}=0.01 \mathrm{mg} / \mathrm{kg} \\
\mathrm{LOQ}=0.05 \mathrm{mg} / \mathrm{kg}\end{array}$ & {$[67]$} \\
\hline $\begin{array}{l}\text { Carminic acid, sunset yellow, } \\
\text { tartrazine }\end{array}$ & $\begin{array}{l}\text { Non-alcoholic } \\
\text { drinks, sweets, } \\
\text { jellies }\end{array}$ & $\begin{array}{l}\text { Capillary } \\
\text { Electrophoresis } \\
\text { (CE) }\end{array}$ & $\begin{array}{c}\mathrm{UV}(\lambda=220 \mathrm{~nm}) \\
36 \mathrm{~cm} \text { capillary; } \\
\text { Separation voltage } \\
(8 \mathrm{kV},-8 \mathrm{kV})\end{array}$ & $\begin{array}{c}\text { Fused-silica } \\
\text { capillaries of } \\
375 \text { od } \mu \mathrm{m} \text { and } \\
75 \mu \mathrm{m} \text { i.d }\end{array}$ & - & $\begin{array}{c}30 \mathrm{mM} \text { PBS buffer (pH6), } \\
\text { with } 0.9 \mathrm{mg} / \mathrm{mL} \text { dASNPs } \\
\text { and } 2 \mathrm{mM} \beta \text {-cyclodextrin } \\
\text { (CD) }\end{array}$ & $\begin{array}{l}\text { Electrokinetic } \\
\text { injection }\end{array}$ & $\begin{array}{c}\mathrm{LOD}=0.03-0.072 \\
\mathrm{mg} / \mathrm{L} \\
\mathrm{LOQ}=0.16-0.31 \\
\mathrm{mg} / \mathrm{L} \\
\end{array}$ & [68] \\
\hline $\begin{array}{l}\text { Amaranth, Ponceau 4R, Sunset } \\
\text { yellow, tartrazine, Sudan I-IV }\end{array}$ & $\begin{array}{l}\text { Soft drinks/solid } \\
\text { samples }\end{array}$ & HPLC-ESI (+)-MS & ESI (+)-MS & $\begin{array}{l}\text { Spherigel C18 } \\
(250 \times 4.6 \mathrm{~mm}, \\
5 \mu \mathrm{m})\end{array}$ & gradient & $\begin{array}{c}\text { aq. methanol } 0.1 \% \\
\text { HCOOH/aqueous } \\
\text { methanol } 20 \mathrm{mM} \\
\mathrm{CH}_{3} \mathrm{COONH} / 1 \% \\
\mathrm{CH}_{3} \mathrm{COOH}\end{array}$ & $20 \mu \mathrm{L}$ & $\begin{array}{l}\mathrm{LOD}=2.0-3.5 \mathrm{ng} \\
\mathrm{LOQ}=5.4-10.5 \mathrm{ng}\end{array}$ & [69] \\
\hline $\begin{array}{l}\text { Brilliant blue, allura red, } \\
\text { amaranth, Erythrosine, ponceau } \\
4 \mathrm{R} \text {, sunset yellow, tartrazine }\end{array}$ & $\begin{array}{l}\text { Soft drinks and } \\
\text { processed meats }\end{array}$ & HPLC & $\begin{array}{c}\text { DAD } \\
\text { Various wavelengths }\end{array}$ & $\begin{array}{l}\text { Inertsil ODS-SP } \\
\text { column } \\
(250 \times 4.6 \mathrm{~mm} \\
5 \mu \mathrm{m}) \\
\end{array}$ & Gradient & $\begin{array}{c}\mathrm{CH}_{3} \mathrm{COONH}_{4}(0.1 \mathrm{M}, \mathrm{pH} \\
= \\
\text { 7.2)-methanol-acetonitrile } \\
(9: 1 v / v) \\
\end{array}$ & $20 \mu \mathrm{L}$ & $\begin{array}{l}\text { LOD: } 0.005 \mu \mathrm{g} / \mathrm{mL} \\
\text { LOQ: } 0.018 \mu \mathrm{g} / \mathrm{mL}\end{array}$ & {$[70]$} \\
\hline
\end{tabular}


Table 3. Cont.

\begin{tabular}{|c|c|c|c|c|c|c|c|c|c|}
\hline $\begin{array}{c}\text { Food } \\
\text { Colorant }\end{array}$ & MATRIX & $\begin{array}{l}\text { Analytical } \\
\text { Technique }\end{array}$ & Detection & Column & Elution & Mobile Phase & Inj. Volume & $\begin{array}{l}\text { Figures of Merit } \\
\text { (LOD, LOQ) }\end{array}$ & Ref. \\
\hline $\begin{array}{l}\text { Brilliant blue, sunset yellow, } \\
\text { tartrazine }\end{array}$ & $\begin{array}{l}\text { Dairy powders, } \\
\text { beverages, } \\
\text { jellies, candies, } \\
\text { syrups, extracts }\end{array}$ & $\begin{array}{l}\text { Flow injection } \\
\text { (FIA) }\end{array}$ & $\begin{array}{c}\text { Amperometric } \\
\text { detection } \\
\text { (boron-doped } \\
\text { diamondelectrode) } \\
\end{array}$ & - & - & $\begin{array}{c}\mathrm{E}_{\text {det. }} 2=-450 \mathrm{mV}(100 \mathrm{~ms} \\
\text { duration) vs. } \\
\mathrm{Ag} / \mathrm{AgCl} \\
(3.0 \mathrm{M} \mathrm{KCl}) .\end{array}$ & & $\mathrm{LOD}=0.8-3.5 \mu \mathrm{M}$ & [71] \\
\hline $\begin{array}{l}\text { Brilliant blue, sunset yellow, } \\
\text { tartrazine }\end{array}$ & $\begin{array}{l}\text { Beverages, } \\
\text { jellies, candies, } \\
\text { condiments, } \\
\text { icings, syrups }\end{array}$ & $\begin{array}{l}\text { Differential pulse } \\
\text { voltammetry } \\
\text { (DPV) }\end{array}$ & $\begin{array}{l}\text { Cathodically } \\
\text { pretreated } \\
\text { boron-doped } \\
\text { diamond (BDD) } \\
\text { elctrode }\end{array}$ & - & - & $\begin{array}{l}30 \mathrm{~Hz} \text {; amplitude(a), } \\
40 \mathrm{mV} \text {; for DPV scan rate } \\
\text { (v), } 0 \mathrm{mVs}\end{array}$ & - & $\mathrm{LOD}=13.1-143 \mathrm{nM}$ & [72] \\
\hline $\begin{array}{l}\text { Erythrosine, carmoisine, } \\
\text { amaranth, ponceau } 4 R \text {, Red 3G }\end{array}$ & Syrups & $\begin{array}{c}\text { Capillary } \\
\text { electrophoresis } \\
\text { (CE) }\end{array}$ & $\begin{array}{c}\text { Laser-induced } \\
\text { fluorescence } \\
\text { detection } \\
\text { Various } \lambda_{\text {exc. }} \lambda_{\text {emis. }} \\
\text { (Nd:YAG laser with } \\
\text { wavelength } 532 \mathrm{~nm} \\
\text { and power } \\
5 \mathrm{~mW} \text { ) }\end{array}$ & $\begin{array}{c}\text { Fused } \\
\text { silica capillary } \\
\text { with I.D. } 50 \mu \mathrm{m} \text {, } \\
\text { O.D. } 360 \mu \mathrm{m}, \\
\text { length } 30 \mathrm{~cm}\end{array}$ & $\mathrm{n} / \mathrm{a}$ & $\begin{array}{l}\mathrm{V}=+17 \mathrm{kV} \\
\text { (intensity of electrical } \\
\text { field was } 460 \mathrm{Vcm}^{-1} \text { ). }\end{array}$ & $\mathrm{n} / \mathrm{a}$ & $\mathrm{LOD}=0.2-0.4 \mu \mathrm{g} / \mathrm{mL}$ & [73] \\
\hline $\begin{array}{c}\text { Tartrazine, sunset yellow, } \\
\text { azorrubine, bordeaux } S \text {, ponceau } \\
4 R \text {, erytrosine, red no } 40 \text {, patent } \\
\text { blue } V \text {, indigo carmin, brilliant } \\
\text { blue }\end{array}$ & $\begin{array}{l}\text { Alcoholic } \\
\text { beverages }\end{array}$ & $\mathrm{CE}$ & $\begin{array}{c}\text { UV/vis } \\
\text { PBS } 10 \mathrm{mM} \\
\text { with sodium dodecyl } \\
\text { sulfate } 10 \mathrm{mM} \text {, } \\
\text { pH 11, and }+25 \mathrm{kV} \text { of } \\
\text { voltage }\end{array}$ & $\begin{array}{c}\text { Fused silica } \\
\text { capillary }(73 \mathrm{~cm})\end{array}$ & $\mathrm{n} / \mathrm{a}$ & $\begin{array}{c}\text { Phosphate buffer solution } \\
\text { of } 10 \mathrm{mM} \\
\text { with sodium dodecyl } \\
\text { sulfate } 10 \mathrm{mM} \text {, } \\
\text { pH } 11 \text {, and }+25 \mathrm{kV} \\
\text { voltage }\end{array}$ & $\mathrm{n} / \mathrm{a}$ & $\begin{array}{c}\mathrm{LOD}=0.4-2.5 \mu \mathrm{g} / \mathrm{mL} \\
\mathrm{LOQ}=\mu \mathrm{g} / \mathrm{mL}\end{array}$ & [74] \\
\hline $\begin{array}{l}\text { Tartazine, Amaranth, Sunset } \\
\text { yellow, allura red, Lutein, } \\
\text { lycopene, } \beta \text {-carotene }\end{array}$ & $\begin{array}{l}\text { Various } \\
\text { foodstuff }\end{array}$ & HPLC & $\begin{array}{c}\text { DAD } \\
\text { Various wavelengths }\end{array}$ & $\begin{array}{c}\text { C18 column } \\
(250 \mathrm{~mm} \times 4.6 \mathrm{~mm} \\
5 \mu \mathrm{m})\end{array}$ & Gradient & $\begin{array}{l}1 \% \mathrm{CH}_{3} \mathrm{COONH}_{4} \\
\text { methanol and acetone; }\end{array}$ & $20 \mu \mathrm{L}$ & $\mathrm{LOD}=0.2-50 \mathrm{ng} / \mathrm{mL}$ & [75] \\
\hline $\begin{array}{l}\text { Tartrazine, Quinoline yellow, } \\
\text { Sunset } \\
\text { Yellow, Carmoisine, Brilliant } \\
\text { Blue }\end{array}$ & Solid foods & $\begin{array}{l}\text { spectrophotometric } \\
\text { method }\end{array}$ & $\begin{array}{c}\text { UV-Vis } \\
\text { Various wavelengths }\end{array}$ & $\mathrm{n} / \mathrm{a}$ & n/a & $\mathrm{n} / \mathrm{a}$ & $\mathrm{n} / \mathrm{a}$ & $\mathrm{LOQ}=1-5 \mu \mathrm{g} / \mathrm{mL}$ & [76] \\
\hline Allura red & Liquid foods & $\begin{array}{l}\text { spectrophotometric } \\
\text { method }\end{array}$ & $\begin{array}{l}\text { UV-Vis } \\
(506 \mathrm{~nm})\end{array}$ & $\mathrm{n} / \mathrm{a}$ & $\mathrm{n} / \mathrm{a}$ & $\mathrm{n} / \mathrm{a}$ & $\mathrm{n} / \mathrm{a}$ & $\mathrm{LOD}=2.35 \mu \mathrm{g} / \mathrm{L}$ & [77] \\
\hline
\end{tabular}


Table 3. Cont.

\begin{tabular}{|c|c|c|c|c|c|c|c|c|c|}
\hline $\begin{array}{c}\text { Food } \\
\text { Colorant }\end{array}$ & MATRIX & $\begin{array}{l}\text { Analytical } \\
\text { Technique }\end{array}$ & Detection & Column & Elution & Mobile Phase & Inj. Volume & $\begin{array}{l}\text { Figures of Merit } \\
\text { (LOD, LOQ) }\end{array}$ & Ref. \\
\hline $\begin{array}{l}\text { Tartrazine, New red, Amaranth, } \\
\text { Ponceau } 4 \mathrm{R}, \text { Sunset yellow, } \\
\text { Allura red, Acid red, Brilliant } \\
\text { Blue, Acid red, Erythrosine, Acid } \\
\text { orange, Basic flavine O, Basic } \\
\text { orange, Siperse blue 106, Crystal } \\
\text { violet, Leucine malachite green, } \\
\text { Leucine crystal violet }\end{array}$ & Meat & UHPLC & $\begin{array}{c}\text { DAD } \\
(200-800 \mathrm{~nm})\end{array}$ & $\begin{array}{l}\text { C18 column } \\
(2.1 \mathrm{~mm} \times 50 \mathrm{~mm} \\
1.7 \mu \mathrm{m})\end{array}$ & Gradient & $\begin{array}{c}\text { (A) } 20 \mathrm{mM}^{-} \\
\mathrm{CH}_{3} \mathrm{COONH} \mathrm{H}_{4}-0.02 \% \\
\text { acetic acid }(\mathrm{pH} 5) \text { and } \\
\text { (B) acetonitrile }\end{array}$ & $2 \mu \mathrm{L}$ & $\begin{array}{c}\mathrm{LOD}=0.96-2.16 \\
\mu \mathrm{g} / \mathrm{kg} \\
\mathrm{LOQ}=1.61-7.19 \\
\mu \mathrm{g} / \mathrm{kg}\end{array}$ & [78] \\
\hline $\begin{array}{l}\text { New red, Amaranth, Camine, } \\
\text { Sunset yellow, Acid Red G, } \\
\text { Allura red, Acid Scarlett GR, } \\
\text { Erythrosine, Rhodamine B, } \\
\text { Sudan I, Para red, Sudan II, } \\
\text { Sudan III, Sudan red 7B, Sudab } \\
\text { IV, Sudan Orange G } \\
\end{array}$ & $\begin{array}{c}\text { Hotpot } \\
\text { condiment }\end{array}$ & HPLC & $\begin{array}{c}\text { DAD } \\
\text { Various wavelengths }\end{array}$ & $\begin{array}{c}\text { C18 column } \\
(4.6 \mathrm{~mm} \times 250 \mathrm{~mm} \\
5 \mu \mathrm{m})\end{array}$ & Gradient & $\begin{array}{l}\text { (A) Methanol; (B) } 0.01 \mathrm{M} \\
\text { PBS ( } \mathrm{pH}=7.5)\end{array}$ & $20 \mu \mathrm{L}$ & $\begin{array}{c}\mathrm{LOD}=0.001-0.003 \\
\mathrm{mg} / \mathrm{kg}\end{array}$ & [79] \\
\hline Allura Red, Ponceau 4R & $\begin{array}{l}\text { Granulated } \\
\text { drinks }\end{array}$ & $\begin{array}{c}\text { UV-visible } \\
\text { spectrophotometer }\end{array}$ & $\begin{array}{l}\text { UV-visible } \\
\text { spectrophotometer } \\
\text { (ZCDS) }\end{array}$ & $\mathrm{n} / \mathrm{a}$ & $\mathrm{n} / \mathrm{a}$ & $\mathrm{n} / \mathrm{a}$ & $\mathrm{n} / \mathrm{a}$ & $\begin{array}{c}\mathrm{LOD}=0.059-0.102 \\
\mu \mathrm{g} / \mathrm{mL} \\
\mathrm{LOQ}=0.198-0.341 \\
\mu \mathrm{g} / \mathrm{mL}\end{array}$ & {$[80]$} \\
\hline $\begin{array}{l}\text { Brilliant Blue, Sunset Yellow, } \\
\text { Tartrazine }\end{array}$ & $\begin{array}{l}\text { Non-alcoholic } \\
\text { drinks, sweets, } \\
\text { jellies }\end{array}$ & HPLC & $\begin{array}{l}\text { UV-Vis } \\
(630 \mathrm{~nm}, 480 \mathrm{~nm}, \\
430 \mathrm{~nm})\end{array}$ & $\begin{array}{c}\text { Modified C18 } \\
\text { column } \\
(250 \times 4.6 \mathrm{~mm}, \\
5 \mu \mathrm{m}) \text { with a } 0.25 \% \\
(v / v) \text { Triton X-100 } \\
\text { aq. solution at } \\
\text { pH 7 } \\
\end{array}$ & Isocratic & $\begin{array}{l}0.25 \mathrm{~mL} \text { of Triton } \mathrm{X}-100 \\
\text { (Sigma) up to } 100 \mathrm{~mL} \\
\text { with } 50 \mathrm{mmol} 1-1 \\
\text { phosphate buffer solution } \\
\text { at pH } 7\end{array}$ & $20 \mu \mathrm{L}$ & $\begin{array}{c}\mathrm{LOD}=0.143-0.080 \\
\mathrm{mg} / \mathrm{L}\end{array}$ & [81] \\
\hline $\begin{array}{l}\text { Tartrazine, Amaranth, Sunset } \\
\text { Yellow, Allura red, Ponceau 4R, } \\
\text { Erythrosine }\end{array}$ & $\begin{array}{l}\text { Soft drinks, } \\
\text { sugar and } \\
\text { gelatin based } \\
\text { confectionery }\end{array}$ & HPLC-UV & $\begin{array}{c}\text { UV } \\
430 \mathrm{~nm}, 510 \mathrm{~nm}\end{array}$ & $\begin{array}{c}\text { C18 column } \\
(250 \mathrm{~mm} \times 4.6 \mathrm{~mm} \\
5 \mu \mathrm{m}\end{array}$ & Gradient & $\begin{array}{c}\text { (A) } 0.1 \mathrm{~mol} / \mathrm{L} \text { ammonium } \\
\text { acetate aqueous solution } \\
\text { (pH 7.5, adjusted with } \\
10 \mathrm{~mol} / \mathrm{L} \text { NaOH- } \\
\text { methanol-acetonitrile } \\
(30: 70, v / v)\end{array}$ & $20 \mu \mathrm{L}$ & $\begin{array}{c}\mathrm{LOD}=0.015-0.32 \\
\mathrm{ng} / \mathrm{mL}\end{array}$ & [82] \\
\hline $\begin{array}{c}\text { Allurea red, Amaranth, } \\
\text { Erythrosine, Ponceau } 4 \mathrm{R} \text {, Sunset } \\
\text { Yellow }\end{array}$ & $\begin{array}{l}\text { Beverages, } \\
\text { alcoholic drinks } \\
\text { and fish foe }\end{array}$ & SERS-Raman & $\begin{array}{l}\text { Radiation of } 514.5 \mathrm{~nm} \\
\text { from an air-cooled } \\
\text { argon ion laser was } \\
\text { used for SERS } \\
\text { excitation }\end{array}$ & $\mathrm{n} / \mathrm{a}$ & $\mathrm{n} / \mathrm{a}$ & $\mathrm{n} / \mathrm{a}$ & $\mathrm{n} / \mathrm{a}$ & $\mathrm{LOD}=10^{-7}-10^{-5} \mathrm{M}$ & [83] \\
\hline Sunset yellow & $\begin{array}{l}\text { beverage, dried } \\
\text { bean curd, } \\
\text { braised pork }\end{array}$ & ELISA & n/a & $\mathrm{n} / \mathrm{a}$ & $\mathrm{n} / \mathrm{a}$ & $\mathrm{n} / \mathrm{a}$ & & $\mathrm{LOD}=25 \mathrm{pg} \mathrm{mL}-1$ & [84] \\
\hline
\end{tabular}


Table 3. Cont.

\begin{tabular}{|c|c|c|c|c|c|c|c|c|c|}
\hline $\begin{array}{c}\text { Food } \\
\text { Colorant }\end{array}$ & MATRIX & $\begin{array}{l}\text { Analytical } \\
\text { Technique }\end{array}$ & Detection & Column & Elution & Mobile Phase & Inj. Volume & $\begin{array}{l}\text { Figures of Merit } \\
\text { (LOD, LOQ) }\end{array}$ & Ref. \\
\hline $\begin{array}{c}\text { (40 food colorants) } \\
\text { Ponceau 6R, Tartrazine, Fast } \\
\text { yellow AB, Amaranth, } \\
\text { Indigotine, Naphthol yellow S, } \\
\text { Chrysoine, Ponceau 4R, Sunset } \\
\text { yellow FCF, Red 10B, Orange G, } \\
\text { Acid violet 7, Brilliant black PN, } \\
\text { Allura red AC, Yellow 2G, Red } \\
\text { 2G, Uranine, Fast red E, Green S, } \\
\text { Ponceau 2R, Azorubine, Orange } \\
\text { I, Quinoline yellow, Martius } \\
\text { yellow, Ponceau SX, Ponceau 3R, } \\
\text { Fast green FCF, Eosine, Brilliant } \\
\text { blue FCF, Orange II, Orange } \\
\text { RN, Acid blue 1, Erythrosine, } \\
\text { Amido black 10B, Acid red 52, } \\
\text { Patent blue V, Acid green 9, } \\
\text { Phloxine B, Benzyl violet 4B, } \\
\text { Rose bengal }\end{array}$ & $\begin{array}{l}\text { Drinks, syrups } \\
\text { and candies }\end{array}$ & HPLC & HPLC-DAD & $\begin{array}{c}\text { C18 column } \\
(50 \mathrm{~mm} \times 4.6 \mathrm{~mm} \\
1.8 \mu \mathrm{m})\end{array}$ & Gradient & $\begin{array}{c}\text { (A) } 0.1 \mathrm{~mol} / \mathrm{L} \text { of } \\
\mathrm{CH}_{3} \mathrm{COONH}{ }_{4} \mathrm{pH} 6.7 \\
\text { and (B) was } \\
\text { Methanol-Acetonitrile } \\
(50: 50, v / v)\end{array}$ & $5 \mu \mathrm{L}$ & $\mathrm{LOD}=0.03-0.1 \mu \mathrm{g} / \mathrm{g}$ & [85] \\
\hline $\begin{array}{c}\text { Ponceau 4R, Sunset Yellow, } \\
\text { Allura Red, Azophloxine, } \\
\text { Ponceauxylidine, Erythrosine, } \\
\text { Orange II }\end{array}$ & $\begin{array}{l}\text { Animal feed } \\
\text { and meat }\end{array}$ & LC & ESI (-)-MS & $\begin{array}{c}\text { C18 column } \\
(2.1 \mathrm{~mm} \times 150 \mathrm{~mm} \\
5 \mu \mathrm{m})\end{array}$ & Gradient & $\begin{array}{l}\text { (A) } 20 \mathrm{mmol} / \mathrm{L} \\
\mathrm{CH}_{3} \mathrm{COONH}_{4} \text { : } \\
\text { Acetonitrile }\end{array}$ & & $\begin{array}{c}\mathrm{LOD}=0.02-21.83 \\
\mathrm{ng} / \mathrm{mL}\end{array}$ & [86] \\
\hline $\begin{array}{c}\text { New Coccine, Indigo Carmine, } \\
\text { Erythrosine, Tartrazine, Sunset } \\
\text { Yellow FCF, Fast Green FCF, } \\
\text { Brilliant Blue FCF, Allura Red } \\
\text { AC, Amaranth, Dimethyl Yellow, } \\
\text { Fast Garnet GBC, Para Red, } \\
\text { Sudan I, Sudan II, Sudan III, } \\
\text { Sudan IV, Sudan Orange G, } \\
\text { Sudan Red 7B, Sudan Red B, } \\
\text { Sudan Red G }\end{array}$ & $\begin{array}{l}\text { Chili powders; } \\
\text { commercial } \\
\text { syrup preserved } \\
\text { fruits }\end{array}$ & LC & $\begin{array}{c}\text { ESI (-) and ESI (+) } \\
\text { MS/MS }\end{array}$ & $\begin{array}{c}\text { Acclaim Polar } \\
\text { Advantage C16 } \\
(3 \mathrm{~mm}, \\
4.6 \times 150 \mathrm{~mm})\end{array}$ & Gradient & $\begin{array}{c}\text { (A) Acetonitrile and (B) } \\
20 \mathrm{mM} \mathrm{CH}_{3} \mathrm{COONH} \\
-1.0 \% \mathrm{CH}_{3} \mathrm{COOH}\end{array}$ & & $\mathrm{LOQ}=0.005-1 \mathrm{mg} / \mathrm{kg}$ & [87] \\
\hline $\begin{array}{l}\text { Multi-class } \\
\text { (53 food colorants) }\end{array}$ & Spices & UHPLC & $\begin{array}{c}\text { QTOF-MS } \\
\text { (sequential window } \\
\text { acquisition of all } \\
\text { theoretical } \\
\text { fragment-ion spectra- } \\
\text { SWATH) }\end{array}$ & $\begin{array}{c}\text { Acquity UPLC } \\
\text { BEH C18 column } \\
(2.1 \times 100 \mathrm{~mm}, \\
1.7 \mu \mathrm{m},)\end{array}$ & Gradient & $\begin{array}{c}\text { (A) Acetonitrile and (B) } \\
10 \mathrm{mM} \mathrm{CH}_{3} \mathrm{COONH}_{4} \\
\mathrm{pH}=6.7\end{array}$ & $\mathrm{n} / \mathrm{a}$ & $\mathrm{n} / \mathrm{a}$ & [88] \\
\hline $\begin{array}{l}\text { Multi-class } \\
\text { (34 water soluble synthetic food } \\
\text { colorants) }\end{array}$ & $\begin{array}{c}\text { Beverages, } \\
\text { syrup, chewing } \\
\text { gum }\end{array}$ & HPLC & $\begin{array}{l}\text { DAD-IT-TOF/MS } \\
(\lambda=200-700 \mathrm{~nm})\end{array}$ & $\begin{array}{c}\text { Atlantis } s^{\mathrm{TM}} \mathrm{dC} 18 \\
(4.6 \mathrm{~mm} \times 250 \mathrm{~mm} \\
5 \mu \mathrm{m})\end{array}$ & Gradient & $\begin{array}{c}\text { (A)20 } \mathrm{mM} \mathrm{HCOONH}_{4} \\
\text { buffer; (B) } \\
\text { methanol/acetonitrile } \\
(1: 1 \mathrm{v} / \mathrm{v})\end{array}$ & $20 \mu \mathrm{L}$ & $\begin{array}{c}\text { LODs }=0.009-0.102 \\
\mu \mathrm{g} / \mathrm{mL} ; \\
\text { LOQs }=0.045-0.203 \\
\mu \mathrm{g} / \mathrm{mL}\end{array}$ & [89] \\
\hline
\end{tabular}


The applied analytical techniques are followed by proper detection approaches. In this framework, simple detector UV-Vis/DAD is mostly applied, followed by MS/MS detectors, UV-Vis spectrometry, and electrochemical detection. The UV-Vis/DAD detection wavelengths depend on the analyte color (i.e., blue, yellow, red) set in any case in the maximum absorbance.

Regarding the MS, listed and EU-approved food colorants could be analyzed in the negative ionization, while for other substances (i.e., Sudan I-IV) positive ionization is applied.

From observation among the available methods of analysis (Table 3 and Figure 3), it could be concluded that traditional TLC methods require a significant sample preparation step and a time-consuming analytical procedure. On the other hand, the HPLC methods need longer analysis time, compared to the respective LC-MS/MS methods, in order to obtain good separation for the same number of analytes [87-89].

As reported recently by Periat et al., full-scan screening methods using HR-MS (High Resolution Mass Spectrometry) have proven to be an alternative to triple quadrupole methods as they could maximize the number of control and analyzed target colorants. Main advantages of the HR-MS can be the reduced sample preparation and the combined targeted analysis with untargeted screening of food colorants with high MS resolving power. Quadropole Time-of-Flight (QTOF) used by Li et al. and by Periat et al. for the detection and identification of coloring compounds in spices provided not only mass accuracy but also MS/MS spectra information and thus increased selectivity. A drawback of the approach could be the high cost of the instrumentation $[85,86]$. As reported by Li et al., HR-MS accurate mass measurements can detect a large number of target analytes, avoiding isobaric interferences in complex samples [89]. A combination of an ESI (or APCI) ionization with an anion trap analyzer linked to a TOF mass analyzer (ESI/APCI-IT-TOF/MS) provides simultaneously multi tandem MS (up to $\mathrm{MS}^{2}$ ) with respective mass accuracy. Currently, there is an increasing interest on the fragmentation mechanism of synthetic food dyes; use of ESI-IT-TOF/MS ${ }^{n}$ in positive as well as in negative ionization modes [87-89] has been increased.

\subsection{Sample Preparation for the Determination of Synthetic Colorants in Foods}

Currently, there is no generally accepted/standard method for synthetic colorant extraction in laboratories. Nevertheless, most extraction procedures follow a common approach, which normally involves firstly the release of desired analytes from their matrices, followed afterwards by removal of extraneous matter/interferences by applying an efficient extraction protocol (i.e., solid-liquid or liquid-liquid extraction) [90].

The applied sample preparation protocols are strongly dependent on the type and nature of the food sample. A short description of the sample preparation protocols, along with their application to the clean-up of food samples, for the analysis of synthetic food colorants is given in Table 4 .

Membrane filtration involves the permeation of the analyte through a thin layer of material. Explicitly, in case of beverages, when filtration is involved, a degassing step needs to be done in advance, in order to remove $\mathrm{CO}_{2}[90]$.

Solid phase extraction (SPE) is one of the most commonly used techniques in determination of food colorants, presenting certain advantages such as simplicity. Polyamide resin used for SPE cleanup retains polar compounds with chemical groups that can be protonated. In acidic $\mathrm{pH}$, during SPE, the colorants are adsorbed to the polyamide stationary phase mainly by Van der Waals interactions. Other hydrophilic substances can mask SPE interaction sites by reducing their binding power for the colorants and consequently reducing the capacity of the cartridges. Some substances, such as amaranth, are strongly retained by SPE cartridges, and the ammonia solution used for elution could be insufficient for its release (low recoveries). 
Table 4. Sample preparation techniques for the determination of synthetic food colorants in food samples.

\begin{tabular}{|c|c|c|c|}
\hline Food Colorant & MATRIX & Extraction/Sample Preparation & Ref. \\
\hline $\begin{array}{l}\text { Amaranth, Ponceau 4R, Sunset Yellow, } \\
\text { Tartrazine and Brilliant Blue }\end{array}$ & & $\begin{array}{l}\text { Reduction of iron (III) in sodium } \\
\text { acetate/hydrochloric acid solution (pH 1.71) } \\
\text { followed by a chromogenic reaction with } \\
\text { potassium hexacyanoferrate (III) to form the } \\
\text { Prussian blue species }\end{array}$ & [9] \\
\hline Sudan I & $\begin{array}{l}\text { Non-alcoholic drinks, } \\
\text { sweets, jellies }\end{array}$ & $\begin{array}{l}\text { Extraction, sonication, centrifugation, filtration. } \\
\text { Sample extracts or liquid samples were filtrated }\end{array}$ & [29] \\
\hline $\begin{array}{l}\text { Tartrazine, Sunset yellow, } \\
\text { Brilliant Blue, Acid red }\end{array}$ & Powder & $\begin{array}{l}\text { Fabrication of flower-like silver nanostructures } \\
\text { by adding } 10 \mathrm{~mL} \text { of ultrapure water, } 2 \mathrm{~mL} \text { of } \\
\text { PVP solution }(1 \%) \text { and } 0.2 \mathrm{~mL} \text { of silver nitrate } \\
\text { solution }(1 \mathrm{~mol} / \mathrm{L})\end{array}$ & [50] \\
\hline $\begin{array}{l}\text { Azorubine, amaranth, cochineal red A, red } \\
2 \mathrm{G} \text {, allura red, azocarmine B (AZO B), } \\
\text { azocarmine G (AZO G), ponceau } 2 \mathrm{R} \text {, } \\
\text { ponceau } 6 \mathrm{R} \text {, tartrazine, sunset yellow, } \\
\text { quinoline yellow, orange II, metanil yellow } \\
\text { (MY), patent blue V, indigo carmine and } \\
\text { brilliant blue FCF. }\end{array}$ & $\begin{array}{l}\text { Solid food/liquid } \\
\text { beverages }\end{array}$ & $\begin{array}{l}4 \text { g solid sample }+20 \mathrm{~mL} \text { ethanol- } \mathrm{H}_{2} \mathrm{O}(1: 1 \mathrm{v} / v) \text {, } \\
\text { ultrasound and shaking, centrifugation, } \\
\text { separation, and SPE in polyamide (PA) } \\
\text { cartridge } \\
\text { Beverages: degas (ultrasound), diluted } 1.1 \text { with } \\
\mathrm{H}_{2} \mathrm{O} \text {, centrifuge }\end{array}$ & [52] \\
\hline $\begin{array}{l}\text { Brilliant Blue FCF, Tartrazine, Amaranth, } \\
\text { Carmine, Sunset yellow, Allura red, } \\
\text { Erythrosine }\end{array}$ & Wine and soft drinks & $\begin{array}{l}\text { Magnetic dispersive solid-phase extraction } \\
\text { (M-dSPE): }\end{array}$ & [53] \\
\hline Allura red & Beverage & $\begin{array}{l}\text { filtered through } 0.45 \mu \mathrm{m} \text { nylon filter and } \\
\text { diluted 1:20 }(v / v) \text { in ultrapure water }\end{array}$ & {$[58,59]$} \\
\hline $\begin{array}{l}\text { Brilliant blue, tartrazine, allura red } \\
\text { Amaranth, Azorubine, Patent Blue V, } \\
\text { Ponceau } 4 \mathrm{R}\end{array}$ & Various food products & $\begin{array}{l}\text { Beverages: sample sonicated, addition of aq. } \\
\mathrm{NH}_{3} \text {, filtration; } \\
\text { Solid: homogenization, addition of aq. } \mathrm{NH}_{3} \text {, } \\
\text { sonication, centrifugation }\end{array}$ & [60] \\
\hline $\begin{array}{l}\text { Brilliant Blue FCF, Indigo carmine, Allura } \\
\text { red, carminic acid, Ponceau } 4 \mathrm{R} \text {, Sunset } \\
\text { yellow, tartrazine }\end{array}$ & $\begin{array}{l}\text { Dairy powders, color } \\
\text { beverages, jellies, } \\
\text { candies, condiments, } \\
\text { icings, syrups, extracts }\end{array}$ & $\begin{array}{l}\text { Flavored milk samples: diluted with ethanol } \\
(1: 1 \mathrm{v} / v) \text {, SPE with PA cartridge }\end{array}$ & [61] \\
\hline $\begin{array}{l}\text { Brilliant Blue FCF, Indigo carmine, } \\
\text { Allura red, carminic acid, Ponceau } 4 \mathrm{R}, \\
\text { Sunset yellow, tartrazine, fast green FCF }\end{array}$ & Liquid foods & $\begin{array}{l}\text { Beverages: degas (ultrasound) and } \\
\text { directly to CE } \\
\text { Milk: diluted with ethanol }(1: 1 v / v), \text { SPE with } \\
\text { PA cartridge } \\
\text { Jelly: blended with ethanol: } \mathrm{H}_{2} \mathrm{O} 1: 1 v / v \text { at } \\
65^{\circ} \mathrm{C} \times 4 \mathrm{~h}+\mathrm{SPE} \text { (Polyamide cartridge) }\end{array}$ & [62] \\
\hline $\begin{array}{l}\text { Sunset yellow, Carmoisine, Amaranth, } \\
\text { Ponceau } 4 \mathrm{R} \text {, Erythrosine., Red } 2 \mathrm{G}, \\
\text { Allura red }\end{array}$ & Soft drinks & $\begin{array}{l}\text { Extraction stage, followed by sonification, } \\
\text { centrifugation, and concentration step + clean } \\
\text { up via SPE on polyamide (PA) cartridges }\end{array}$ & [63] \\
\hline $\begin{array}{l}\text { Tartrazine, Quinoline Yellow, Sunset Yellow, } \\
\text { Carmoisine, Ponceau, 4R, Allura Red, } \\
\text { Indigo Carmine, Brilliant Blue }\end{array}$ & $\begin{array}{l}\text { Various foods and } \\
\text { medicines }\end{array}$ & Homogenization, dissolution, filtration & [64] \\
\hline
\end{tabular}


Table 4. Cont.

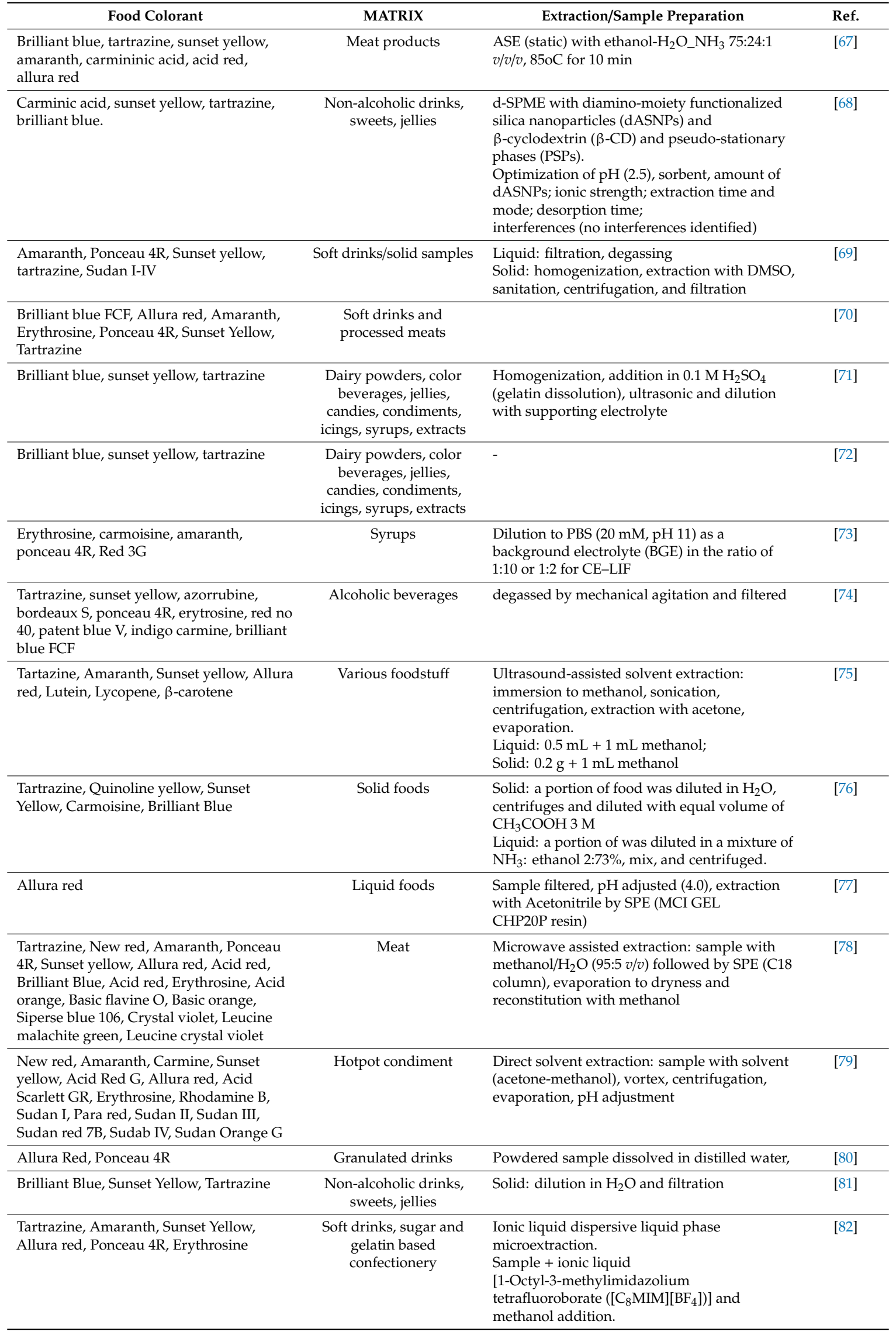


Table 4. Cont.

\begin{tabular}{|c|c|c|c|}
\hline Food Colorant & MATRIX & Extraction/Sample Preparation & Ref. \\
\hline $\begin{array}{l}\text { Allura red, Amaranth, Erythrosine, } \\
\text { Ponceau 4R, Sunset Yellow }\end{array}$ & $\begin{array}{l}\text { Beverages, alcoholic } \\
\text { drinks and fish foe }\end{array}$ & Synthesis of the G/Ag nanoparticle composite & [83] \\
\hline $\begin{array}{l}\text { (40 food colorants) Ponceau 6R, Tartrazine, } \\
\text { Fast yellow AB, Amaranth, Indigotine, } \\
\text { Naphthol yellow S, Chrysoine, Ponceau 4R, } \\
\text { Sunset yellow FCF, Red 10B, Orange G, } \\
\text { Acid violet 7, Brilliant black PN, Allura red } \\
\text { AC, Yellow 2G, Red 2G, Uranine, Fast red E, } \\
\text { Green S, Ponceau 2R, Azorubine, Orange I, } \\
\text { Quinoline yellow, Martius yellow, Ponceau } \\
\text { SX, Ponceau 3R, Fast green FCF, Eosine, } \\
\text { Brilliant blue FCF, Orange II, Orange } \\
\text { RN, Acid blue 1, Erythrosine, Amido black } \\
\text { 10B, Acid red 52, Patent blue V, Acid green } \\
\text { 9, Phloxine B, Benzyl violet 4B, Rose bengal. }\end{array}$ & $\begin{array}{l}\text { Drinks, syrups, and } \\
\text { candies }\end{array}$ & $\begin{array}{l}\text { Drinks: degas, evaporation } \\
\text { Solid: grind, mixing with solvent and SPE with } \\
\text { PA column with } 1 \% \mathrm{NH}_{3} / \text { ethanol solution }\end{array}$ & [85] \\
\hline $\begin{array}{l}\text { Ponceau 4R, Sunset Yellow, Allura Red, } \\
\text { Azophloxine, Ponceauxylidine, Erythrosine } \\
\text { Orange II }\end{array}$ & Animal feed and meat & $\begin{array}{l}\text { Homogenization, extraction with } \\
\text { ethanol: } \mathrm{NH}_{3}: \mathrm{H}_{2} \mathrm{O}(80: 1: 19 v / v) \text {, } \\
\text { evaporation, reconstitution }\end{array}$ & [86] \\
\hline $\begin{array}{l}\text { New Coccine, Indigo Carmine, Erythrosine, } \\
\text { Tartrazine, Sunset Yellow FCF, Fast Green } \\
\text { FCF, Brilliant Blue FCF, Allura Red AC, } \\
\text { Amaranth, Dimethyl Yellow, Fast Garnet } \\
\text { GBC, Para Red, Sudan I, Sudan II, Sudan III, } \\
\text { Sudan IV, Sudan Orange G, Sudan Red 7B, } \\
\text { Sudan Red B, Sudan Red G }\end{array}$ & $\begin{array}{l}\text { Chili powders; } \\
\text { commercial syrup } \\
\text { preserved fruits }\end{array}$ & $\begin{array}{l}\text { Homogenization and extraction with } \\
\text { Acetonitrile twice }\end{array}$ & [87] \\
\hline $\begin{array}{l}\text { Multi-class } \\
\text { (53 food colorants) }\end{array}$ & Spices & $\begin{array}{l}\text { Extraction with } \\
\mathrm{H}_{2} \mathrm{O} / \text { methanol/acetonitrile/THF, 9:1:5:5, v/v/v/v) }\end{array}$ & [88] \\
\hline
\end{tabular}

Dispersive solid phase extraction (d-SPE) analysis is a simple sample preparation methodology that is suitable for a wide variety of food and agricultural products, as is also QuEChERS, introduced for pesticides from Anastassiades et al. [91]. In case of synthetic colorants, a modified QuEChERS method has been reported (magnetic-dSPE) using cross-linking magnetic polymer $\left(\mathrm{NH}_{2}\right.$-LDC-MP) containing less hydrophilic amino groups and more lipophilic styrene monomer for cleaning up the synthetic food colorants from wine and soft drinks [53].

Liquid-liquid extraction (LLE) deals with the separation of substances based on their relative solubility in two different immiscible liquids. Common solvents for the extraction of synthetic food colorants from food matrices are water, ethanol, methanol, isopropyl alcohol, ammoniac ethanol, ethyl acetate, ammonia, cyclohexane, and tetra-n-butyl ammonium phosphate. Wu et al. has also reported an extraction method based on Ionic liquid dispersive liquid phase microextraction using the ionic liquid (1-Octyl-3-methylimidazolium tetrafluoroborate $\left.\left(\left(\mathrm{C}_{8} \mathrm{MIM}\right)\left(\mathrm{BF}_{4}\right)\right)\right)$ [81].

In the literature, a limited number of protocols exists dealing with other types of extraction methods for synthetic food colorants, such as MAE and Ultrasound Assisted Extraction (UAE). These kinds of extractions require special instrumentation and most probably can be beneficial for a laboratory, as extractions with organic solvents are characterized by consumption of high volumes of solvents, are time consuming, and in some cases have low recoveries [90].

\section{Conclusions}

The use of food colorants in the production of foods leads to the need for the development of accurate, precise, sensitive, and selective analytical methods for their analysis and quantification. Certain interest in the impacts of food colorants is being reported worldwide. There is a plethora of analytical research works that deal with the analytical challenge of the analysis and quantification 
of either natural or synthetic food colorants. The research community gives more attention to the appropriate analysis, in sufficient concentration or mass fraction levels, mostly to synthetic food colorants rather than natural ones.

Analytical methodologies have much more to offer in this direction and, as it could be concluded from synthetic colorants, HPLC is the most frequently used followed by capillary electrophoresis. In terms of detection methods, the simple UV-Vis/DAD is the predominant one followed by tandem MS. The analytical techniques and sample preparation methodologies presented cover the existing methodologies mainly applied during the last decade.

Regarding sample preparation, this is highly sample dependent. It could involve the application of different extraction techniques, such as membrane filtration, liquid-liquid and solid phase extraction techniques, for cleaning-up the highly complex matrix of food products. Sample preparation is of great importance and must be carefully developed, in order to avoid or eliminate existing matrix interferences aiming to the development of simple, selective, and precise methods of extraction.

In the case of simple liquid samples, dilution and injection are preferred, though in other cases such as high protein content foods, specific steps need to be followed for sufficient sample clean-up.

Author Contributions: Conceptualization, E.T., H.G., and K.N.; methodology, K.N.; formal analysis, E.T. and K.N.; investigation, K.N.; resources, E.T. and K.N.; data curation, E.T.; writing一 original draft preparation, K.N.; writing-review and editing, H.G. and E.T.; visualization, E.T.; supervision, E.T.; project administration, E.T. All authors have read and agreed to the published version of the manuscript.

Funding: This research received no external funding.

Acknowledgments: We acknowledge support of this work by the project "FoodOmicsGR Comprehensive Characterisation of Foods" (MIS 5029057) which is implemented under the Action "Reinforcement of the Research and Innovation Infrastructure", funded by the Operational Programme Competitiveness, Entrepreneurship and Innovation (NSRF2014-2020) and co-financed by Greece and the European Union (European Regional Development Fund).

Conflicts of Interest: The authors declare no conflict of interest.

\section{References}

1. Codex Alimentarius. Available online: http://www.codexalimentarius.org/standards/gsfa/ (accessed on 18 September 2018).

2. Carocho, M.; Morales, P.; Ferreira, I.C.F.R. Natural food additives: Quo vadis? Trans Food Sci. Technol. 2015, 45, 284-295. [CrossRef]

3. Amchova, P.; Kotolova, H.; Ruda-Kucerova, J. Health safety issues of synthetic food colorants. Reg. Toxic. Pharm. 2015, 73, 914-922. [CrossRef]

4. Aberamound, A. A Review Article on Edible Pigments Properties and Sources as Natural Biocolorants in Foodstuff and Food Industry. World J. Dairy Food Sci. 2011, 6, 71-78.

5. Burrows, A.J.D. Palette of Our Palates: A Brief History of Food Coloring and Its Regulation. Compreh. Rev. Food Sci. Food Saf. 2009, 9, 394-408. [CrossRef]

6. Martins, N.; Roriz, C.L.; Morales, P.; Barros, L.; Ferreira, I.C. Food colorants: Challenges, opportunities and current desires of agro-industries to en-sure consumer expectations and regulatory practices. Trends Food Sci. Technol. 2016, 52, 1-15. [CrossRef]

7. Rodriguez-Amaya, D.B. Natural food pigments and colorants. Curr. Opin. Food Sci. 2016, 7, 20-26. [CrossRef]

8. Tumolo, T.; Lanfer-Marquez, U.M. Copper chlorophyllin: A food colorant with bioactive properties? Food Res. Int. 2012, 46, 451-459. [CrossRef]

9. Ni, Y.; Wang, Y.; Kokot, S. Simultaneous kinetic spectrophotometric analysis of five synthetic food colorants with the aid of chemometrics. Talanta 2009, 78, 432-441. [CrossRef]

10. Sigurdson, G.T.; Tang, P.; Giusti, M.M. Natural colorants: food colorants from natural sources. Annu. Rev. Food Sci. Technol. 2017, 8, 261-280. [CrossRef]

11. Carocho, M.; Barreiro, M.F.; Morales, P.; Ferreira, I.C. Adding molecules to food, pros and cons: A review on synthetic and natural food additives. Compr. Rev. Food Sci. Food Saf. 2014, 13, 377-399. [CrossRef] 
12. Dias, M.I.; Ferreira, I.C.; Barreiro, M.F. Microencapsulation of bioactives for food applications. Food Funct. 2015, 6, 1035-1052. [CrossRef] [PubMed]

13. Patakova, P. Monascus secondary metabolites: Production and biological activity. J. Ind. Microbiol. Biotechnol. 2013, 40, 169-181. [CrossRef] [PubMed]

14. Wang, C.; Chen, D.; Chen, M.; Wang, Y.; Li, Z.; Li, F. Stimulatory effects of blue light on the growth, monascin and ankaflavin production in Monascus. Biotechnol. Lett. 2015, 37, 1043-1048. [CrossRef]

15. Dias, M.G.; Camoes, M.F.G.; Oliveira, L. Carotenoids in traditional Portuguese fruits and vegetables. Food Chem. 2009, 113, 808-815. [CrossRef]

16. Carocho, M.; Ferreira, I.C. A review on antioxidants, prooxidants and related controversy: Natural and synthetic compounds, screening and analysis methodologies and future perspectives. Food Chem. Toxicol. 2013, 51, 15-25. [CrossRef]

17. Masone, D.; Chanforan, C. Study on the interaction of artificial and natural food colorants with human serum albumin: A computational point of view. Comput. Biol. Chem. 2015, 56, 152-158. [CrossRef]

18. Oplatowska-Stachowiak, M.; Elliottt, C.T. Food colors: Existing and emerging food safety concerns. Crit. Rev. Food Sci. Nutr. 2017, 57, 524-548. [CrossRef]

19. Feng, J.; Cerniglia, C.E.; Chen, H. Toxicological significance of azo dye metabolism by human intestinal microbiota. Front. Biosci. 2012, 4, 568-586. [CrossRef]

20. Golka, K.; Kopps, S.; Myslak, Z.W. Carcinogenicity of azo colorants: Influence of solubility and bioavailability. Toxicol. Lett. 2004, 151, 203-210. [CrossRef]

21. Vojdani, A.; Vojdani, C. Immune reactivity to food coloring. Altern. Ther. Health Med. 2015, 21, 52-62.

22. Mpountoukas, P.; Pantazaki, A.; Kostareli, E.; Christodoulou, P.; Kareli, D.; Poliliou, S.; Mourelatos, C.; Lambropoulou, V.; Lialiaris, T. Cytogenetic evaluation and DNA interaction studies of the food colorants amaranth, erythrosine and tartrazine. Food Chem. Toxic. 2010, 48, 2934-2944. [CrossRef]

23. Pan, X.; Qin, P.; Liu, R.; Wang, J. Characterizing the Interaction between tartra-zine and two serum albumins by a hybrid spectroscopic approach. J. Agric. Food Chem. 2011, 59, 6650-6656. [CrossRef] [PubMed]

24. Basu, A.; Kumar, G.S. Study on the interaction of the toxic food additive Carmoisine with serum albumins: A microcalorimetric investigation. J. Hazard Mater. 2014, 273, 200-206. [CrossRef] [PubMed]

25. Datta, S.; Mahapatra, N.; Halder, M. pH-insensitive electrostatic interaction of carmoisine with two serum proteins: A possible caution on its uses in food and pharmaceutical industry. J. Photochem. Photobiol. B 2013, 124, 50-62. [CrossRef] [PubMed]

26. Wang, L.; Zhang, G.; Wang, Y. Binding properties of food colorant Allura Red with human serum albumin in vitro. Mol. Biol. Rep. 2014, 41, 3381-3391. [CrossRef]

27. Wu, D.; Yan, J.; Wang, J.; Wang, Q.; Li, H. Characterisation of interaction between food colourant A Allura red AC and human serum albumin: Multi-spectroscopic analyses and docking simulations. Food Chem. 2015, 170, 423-429. [CrossRef]

28. Tellier, F.; Steibel, J.; Chabrier, R.; Ble, F.X.; Tubaldo, H.; Rasata, R.; Chambron, J.; Duportail, G.; Simon, H.; Rodier, J.F.; et al. Sentinel lymph nodes fluorescence detection and imaging using Patent Blue V bound to human serum albumin. Biomed. Opt. Exp. 2012, 3, 2306-2316. [CrossRef]

29. Wang, Y.; Wei, D.; Yang, H.; Yang, Y.; Xing, W.; Li, Y. Development of a highly sensitive and specific monoclonal antibody-based enzyme-linked immunosorbent assay (ELISA) for detection of Sudan I in food samples. Talanta 2009, 77, 1783-1789. [CrossRef]

30. Moller, P.; Wallin, H. Genotoxic hazards of azo pigments and other colorants related to 1-phenylazo2-hydroxynaphthalene. Mutat. Res. 2000, 462, 13-30. [CrossRef]

31. Stiborova, M.; Martinek, V.; Rydlova, H.; Hodek, P.; Frei, E. Sudan I is a Potential Carcinogen for Humans: Evidence for Its Metabolic Activation and Detoxication by Human Recombinant Cytochrome P450 1A1 and Liver Microsomes. Cancer Res. 2002, 62, 5678-5684.

32. European Food Safety Authority. Available online: http://www.efsa.europa.eu (accessed on 15 September 2018).

33. Lehto, S.; Buchweitz, M.; Klimm, A.; Straßburger, R.; Bechtold, C.; Ulberth, F. Comparison of food colour regulations in the EU and the US: A review of cur-rent provisions. Food Addit. Contam. 2017, 34, 335-355. [CrossRef] [PubMed]

34. Dykes, L.; Rooney, W.L.; Rooney, L.W. Evaluation of phenolics and antioxidant activity of black sorghum hybrids. J. Cereal Sci. 2013, 58, 278-283. [CrossRef] 
35. Sivakumar, V.; Vijaeeswarri, J.; Anna, J.L. Effective natural dye extraction from different plant materials using ultrasound. Ind. Crop. Prod. 2011, 33, 116-122. [CrossRef]

36. Sagdic, O.; Ekici, L.; Ozturk, I.; Tekinay, T.; Polat, B.; Tastemur, B.; Senturk, B. Cytotoxic and bioactive properties of different color tulip flowers and degradation kinetic of tulip flower anthocyanins. Food Chem. Toxicol. 2013, 58, 432-439. [CrossRef] [PubMed]

37. Longo, L.; Scardino, A.; Vasapollo, G. Identification and quantification of anthocyanins in the berries of Pistacia lentiscus L., Phillyrea latifolia L. and Rubia peregrina L. Innov. Food Sci. Emerg. Technol. 2007, 8, 360-364. [CrossRef]

38. Stintzing, F.C.; Schieber, A.; Carle, R. Betacyanins in fruits from red-purple pitaya, Hylocereus polyrhizus (Weber) Britton \& Rose. Food Chem. 2002, 77, 101-106.

39. Ravichandran, K.; Saw, N.M.M.T.; Mohdaly, A.A.; Gabr, A.M.; Kastell, A.; Riedel, H.; Cai, Z.-Z.; Knorr, D.; Smetanska, I. Impact of processing of red beet on betalain content and antioxidant activity. Food Res. Int. 2013, 50, 670-675. [CrossRef]

40. Cassano, A.; Conidi, C.; Drioli, E. Physico-chemical parameters of cactus pear (Opuntia ficus-indica) juice clarified by microfiltration and ultrafiltration processes. Desalination 2010, 250, 1101-1104. [CrossRef]

41. Otalora, M.C.; Carriazo, J.G.; Iturriaga, L.; Nazareno, M.A.; Osorio, C. Micro-encapsulation of betalains obtained from cactus fruit (Opuntia ficus- indica) by spray drying using cactus cladode mucilage and maltodextrin as encapsulating agents. Food Chem. 2015, 187, 174-181. [CrossRef]

42. Khan, M.I.; Giridhar, P. Enhanced chemical stability, chromatic properties and regeneration of betalains in Rivina humilis L. berry juice. LWT_Food Sci. Technol. 2014, 58, 649-657. [CrossRef]

43. Sun, M.; Temelli, F. Supercritical carbon dioxide extraction of carotenoids from carrot using canola oil as a continuous co-solvent. J. Supercrit. Fluids 2006, 37, 397-408. [CrossRef]

44. Sobral, D.; Costa, R.G.B.; Machado, G.M.; Paula, J.C.J.; Teodoro, V.A.M.; Nunes, N.M.; Pinto, M.S. Can lutein replace annatto in the manufacture of Prato cheese? LWT-Food Sci. Technol. 2016, 68, 349-355. [CrossRef]

45. Khalil, M.; Raila, J.; Ali, M.; Islam, K.M.S.; Schenk, R.; Krause, J.-P.; Schweigert, F.H.; Rawel, H. Stability and bioavailability of lutein ester supplements from Tagetes flower prepared under food processing conditions. J. Funct. Foods 2012, 4, 602-610. [CrossRef]

46. Grewe, C.; Menge, S.; Griehl, C. Enantioselective separation of all-E- astaxanthin and its determination in microbial sources. J. Chromatogr. A 2007, 1166, 97-100. [CrossRef] [PubMed]

47. Wada, M.; Kido, H.; Ohyama, K.; Ichibangase, T.; Kishikawa, N.; Ohba, Y.; Nakashima, M.N.; Kuroda, N.; Nakashima, K. Chemiluminescent screening of quenching effects of natural colorants against reactive oxygen species: Evaluation of grape seed, monascus, gardenia and red radish extracts as multi-functional food additives. Food Chem. 2007, 101, 980-986. [CrossRef]

48. Thalhamer, B.; Buchberger, W. Adulteration of beetroot red and paprika extract based food colorant with Monascus red pigments and their detection by HPLC-QTof MS analyses. Food Control 2019, 105, 58-63. [CrossRef]

49. Minioti, K.S.; Sakellariou, C.F.; Thomaidis, N.S. Determination of 13 synthetic food colorants in water-soluble foods by reversed-phase high- performance liquid chromatography coupled with diode-array detector. Anal. Chim. Acta 2007, 583, 103-110. [CrossRef]

50. Ai, Y.-J.; Wu, Y.-X.; Dong, Q.-M.; Li, J.-B.; Xu, B.-J.; Yu, Z.; Ni, D. Rapid qualitative and quantitative determination of food colorants by both T Raman spectra and Surface-enhanced Raman Scattering (SERS). Food Chem. 2018, 241, 427-433. [CrossRef]

51. Al-Degs, Y.S. Determination of three dyes in commercial soft drinks using HLA/GO and liquid chromatography. Food Chem. 2009, 117, 485-490. [CrossRef]

52. Bonan, S.; Fedrizzi, G.; Menotta, S.; Elisabetta, C. Simultaneous determination of synthetic dyes in foodstuffs and beverages by high-performance liquid chromatography coupled with diode-array detector. Dyes Pigment. 2013, 99, 36-40. [CrossRef]

53. Chen, X.H.; Zhao, Y.G.; Shen, H.Y.; Zhou, L.X.; Pan, S.D.; Jin, M.C. Fast determination of seven synthetic pigments from wine and soft drinks using magnetic dispersive solid-phase extraction followed by liquid chromatography-tandem mass spectrometry. J. Chromatogr. A 2014, 1346, 123-128. [CrossRef]

54. De Andrade, F.I.; Guedes, M.I.F.; Vieira, Í.G.; Mendes, F.N.P.; Rodrigues, P.A.S.; Maia, C.S.C.; Ávila, M.M.M.; Ribeiro, L.d.M. Determination of synthetic food dyes in commercial soft drinks by TLC and ion-pair HPLC. Food Chem. 2014, 157, 193-198. [CrossRef] [PubMed] 
55. El-Sheikh, A.H.; Al-Degs, Y.S. Spectrophotometric determination of food dyes in soft drinks by second order multivariate calibration of the absorbance spectra-pH data matrices. Dyes Pigments 2013, 97, 330-339. [CrossRef]

56. Gosetti, F.; Gianotti, V.; Polati, S.; Gennaro, M.C. HPLC-MS degradation study of E110 Sunset Yellow FCF in a commercial beverage. J. Chromatogr. A 2005, 1090, 107-115. [CrossRef] [PubMed]

57. Gosetti, F.; Frascarolo, P.; Mazzucco, E.; Gianotti, V.; Bottaro, M.; Gennaro, M.C. Photodegradation of E110 and E122 dyes in a commercial aperitif: A high performance liquid chromatography-diode array-tandem mass spectrometry study. J. Chromatogr. A 2008, 1202, 58-63. [CrossRef]

58. Gosetti, F.; Chiuminatto, U.; Mazzucco, E.; Calabrese, G.; Gennaro, M.C.; Marengo, E. Identification of photodegradation products of Allura Red AC (E129) in a beverage by ultra-high performance liquid chromatography-quadrupole- time-of-flight mass spectrometry. Anal. Chim. Acta 2012, 746, 84-89. [CrossRef]

59. Gosetti, F.; Chiuminatto, U.; Mazzucco, E.; Calabrese, G.; Gennaro, M.C.; Marengo, E. Non-target screening of Allura Red AC photodegradation products in a beverage through ultra-high performance liquid chromatography coupled with hybrid triple quadrupole/linear ion trap mass spectrometry. Food Chem. 2013, 136, 617-623. [CrossRef]

60. Harp, B.P.; Miranda-Bermudez, E.; Barrows, J.N. Determination of seven certified color additives in food products using liquid chromatography. J. Agric. Food Chem. 2013, 61, 3726-3736. [CrossRef]

61. Huang, H.-Y.; Shih, Y.-C.; Chen, Y.-C. Determining eight colorants in milk beverages by capillary electrophoresis. J. Chromatogr. A 2002, 959, 317-325. [CrossRef]

62. Huang, H.-Y.; Chiu, C.-W.; Sue, S.-L.; Cheng, C.-F. Analysis of food colorants by capillary electrophoresis with large- volume sample stacking. J. Chromatogr. A 2003, 995, 29-36. [CrossRef]

63. Karanikolopoulos, G.; Gerakis, A.; Papadopoulou, K.; Mastrantoni, I. Determination of synthetic food colorants in fish products by an HPLC-DAD method. Food Chem. 2015, 177, 197-203. [CrossRef] [PubMed]

64. Khanavi, M.; Hajimahmoodi, M.; Ranjbar, A.M.; Oveisi, M.R.; Ardekani, M.R.S.; Mogaddam, G. Development of a green chromatographic method for simultaneous determination of food colorants. Food Anal. Methods 2012, 5, 408-415. [CrossRef]

65. Kirschbaum, J.; Krause, C.; Brückner, H. Liquid chromatographic quantification of synthetic colorants in fish roe and caviar. Eur. Food Res. Technol. 2006, 222, 572-579. [CrossRef]

66. Kong, C.; Fodjo, E.K.; Li, D.; Cai, Y.; Huang, D.; Wang, Y.; Shen, X. Chitosan-based ad-sorption and freeze deproteinization: Improved extraction and purifica-tion of synthetic colorants from protein-rich food samples. Food Chem. 2015, 188, 240-247. [CrossRef]

67. Liao, Q.G.; Li, W.H.; Luo, L.G. Applicability of accelerated solvent extraction for synthetic colorants analysis in meat products with ultrahigh performance liquid chromatography-photodiode array detection. Anal. Chim. Acta 2012, 716, 128-132. [CrossRef]

68. Liu, F.-J.; Liu, C.-T.; Li, W.; Tang, A.-N. Dispersive solid-phase micro- extraction and capillary electrophoresis separation of food colorants in beverages using diamino moiety functionalized silica nanoparticles as both extractant and pseudostationary phase. Talanta 2015, 132, 366-372. [CrossRef]

69. Ma, M.; Luo, X.; Chen, B.; Su, S.; Yao, S. Simultaneous determination of water-soluble and fat-soluble synthetic colorants in foodstuff by high- perfo-mance liquid chromatography-diode array detection-electrospray mass spectrometry. J. Chromatogr. A 2006, 1103, 170-176. [CrossRef]

70. Ma, K.; Yang, Y.N.; Jiang, X.X.; Zhao, M.; Cai, Y.Q. Simultaneous determination of 20 food additives by high performance liquid chromatography with photo-diode array detector. Chin. Chem. Lett. 2012, 23, 492-495. [CrossRef]

71. Medeiros, R.A.; Lourencao, B.C.; Rocha-Filho, R.C.; Fatibello-Filho, O. Flow injection simultaneous determination of synthetic colorants in food using multiple pulse amperometric detection with a boron-doped diamond electrode. Talanta 2012, 99, 883-889. [CrossRef]

72. Medeiros, R.A.; Lourencao, B.C.; Rocha-Filho, R.C.; Fatibello-Filho, O. Simultaneous voltammetric determination of synthetic colorants in food using a cathodically pretreated boron-doped diamond electrode. Talanta 2012, 97, 291-297. [CrossRef]

73. Ryvolova, M.; Taborský, P.; Vrabel, P.; Krasenský, P.; Preisler, J. Sensitive determination of erythrosine and other red food colorants using capillary electrophoresis with laser-induced fluorescence detection. J. Chromatogr. A 2007, 1141, 206-211. [CrossRef] [PubMed] 
74. Prado, M.A.; Boas, L.F.V.; Bronze, M.R.; Godoy, H.T. Validation of methodology for simultaneous determination of synthetic dyes in alcoholic beverages by capillary electrophoresis. J. Chromatogr. A 2006, 1136, 231-236. [CrossRef] [PubMed]

75. Shen, Y.; Zhang, X.; Prinyawiwatkul, W.; Xu, Z. Simultaneous determination of red and yellow artificial food colourants and carotenoid pigments in food products. Food Chem. 2014, 157, 553-558. [CrossRef] [PubMed]

76. Sorouraddin, M.H.; Rostami, A.; Saadati, M.A. simple and portable multi-colour light emitting diode based photocolourimeter for the analysis of mixtures of five common food dyes. Food Chem. 2011, 127, 308-313. [CrossRef]

77. Soylak, M.; Unsal, Y.E.; Tuzen, M. Spectrophotometric determination of trace levels of allura red in water samples after separation and pre-concentration. Food Chem. Toxicol. 2011, 49, 1183-1187. [CrossRef]

78. Sun, H.; Sun, N.; Li, H.; Zhang, J.; Yang, Y. Development of multiresidue analysis for 21 synthetic colorants in meat by microwave-assisted extraction-solid-phase extraction-reversed-phase ultrahigh performance liquid chromatography. Food Anal. Methods 2013, 6, 1291-1299. [CrossRef]

79. Tang, B.; Xi, C.; Zou, Y.; Wang, G.; Li, X.; Zhang, L.; Chen, D.; Zhang, J. Simultaneous determination of 16 synthetic colorants in hotpot condiment by high performance liquid chromatography. J. Chromatogr. B 2014, 960, 87-91. [CrossRef]

80. Turak, F.; Ozgur, M.U. Simultaneous determination of allura red and ponceau 4 R in drinks with the use of four derivative spectrophotometric methods and comparison with high-performance liquid chromatography. J. AOAC Int. 2013, 96, 1377-1386. [CrossRef]

81. Vidotti, E.C.; Costa, W.F.; Oliveira, C.C. Development of a green chromatographic method for determination of colorants in food samples. Talanta 2006, 68, 516-521. [CrossRef]

82. Wu, H.; Guo, J.B.; Du, L.M.; Tian, H.; Hao, C.X.; Wang, Z.F.; Wang, J.Y. A rapid shaking-based ionic liquid dispersive liquid phase micro extraction for the simultaneous determination of six synthetic food colourants in soft drinks, sugar-and gelatin-based confectionery by high-performance liquid chromatography. Food Chem. 2013, 141, 182-186. [CrossRef]

83. Xie, Y.; Li, Y.; Niu, L.; Wang, H.; Qian, H.; Yao, W. A novel surface-enhanced Raman scattering sensor to detect prohibited colorants in food by graphene/silver nanocomposite. Talanta 2012, 100, 32-37. [CrossRef] [PubMed]

84. Xing, Y.; Meng, M.; Xue, H.; Zhang, T.; Yin, Y.; Xi, R. Development of a polyclonal antibody-based enzyme-linked immunosorbent assay (ELISA) for detection of sunset yellow FCF in food samples. Talanta 2012, 99, 125-131. [CrossRef] [PubMed]

85. Yoshioka, N.; Ichihashi, K. Determination of 40 synthetic food colors in drinks and candies by highperformance liquid chromatography using a short column with photodiode array detection. Talanta 2008, 74, 1408-1413. [CrossRef] [PubMed]

86. Zou, T.; He, P.; Yasen, A.; Li, Z. Determination of seven synthetic dyes in animal feeds and meat by high performance liquid chromatography with diode array and tandem mass detectors. Food Chem. 2013, 138, 1742-1748. [CrossRef] [PubMed]

87. Tsai, C.-F.; Kuo, C.-H.; Shih, D.Y.-C. Determination of 20 synthetic dyes in chili powders and syrup-preserved fruits by liquid chromatography/tandem mass spectrometry. J. Food Drug Anal. 2015, 23, 453-462. [CrossRef] [PubMed]

88. Périat, A.; Bieri, S.; Mottier, N. SWATH-MS screening strategy for the determination of food dyes in spices by UHPLC-HRMS. Food Chem. X 2019, 1, 100009. [CrossRef]

89. Li, X.Q.; Zhang, Q.H.; Ma, K.; Li, H.M.; Guo, Z. Identification and determination of 34 water-soluble synthetic dyes in foodstuff by high performance liquid chromatography-diode array detection-ion trap time-of-flight tandem mass spectrometry. Food Chem. 2015, 182, 316-326. [CrossRef]

90. Yamjala, K.; Nainar, M.S.; Ramisetti, N.R. Methods for the analysis of azo dyes employed in food industry-A review. Food Chem. 2016, 192, 813-824. [CrossRef]

91. Anastassiades, M.; Lehotay, S.J.; Stajnbaher, D.; Schenck, F.J. Fast and easy multiresidue method employing acetonitrile extraction/partitioning and "dispersive solid-phase extraction" for the determination of pesticide residues in produce. J. AOAC Int. 2003, 86, 412-431.

(C) 2020 by the authors. Licensee MDPI, Basel, Switzerland. This article is an open access article distributed under the terms and conditions of the Creative Commons Attribution (CC BY) license (http://creativecommons.org/licenses/by/4.0/). 